\title{
Synchronization and Stability of a Three Co-Rotating Rotor System Coupled with Springs in a Non-Resonance System
}

\author{
Mingjun Du ${ }^{1},{ }^{*}$ - Yongjun Hou ${ }^{1}$ - Tong Tang 2 - Lian Tang ${ }^{3}$ - Jialong Wang ${ }^{1}-$ Hongbo Gao $^{1}$ \\ ${ }^{1}$ Southwest Petroleum University, School of Mechatronics Engineering, China \\ ${ }^{2}$ AECC Chengdu Engine Company Limited, China \\ ${ }^{3}$ Sichuan Aviation Industry Chuanxi Machinery Company Limited, China
}

With the rapid development of horizontal drilling technology, the drilling fluid shale shaker (DFSS) features high capacity and high efficiency. Hence, a vibrating mechanism of a three co-rotating rotor system coupled with springs is proposed for designing large-sized and heavy-duty vibrating screens in petroleum drilling engineering. To master synchronization of the vibrating system, the dynamic equations of three corotating rotors coupled with springs are first developed based on Lagrange's equations. Second, synchronous conditions of the system are derived based on the average method, and its stability criterion is obtained by adopting Hamilton's principle. Furthermore, the influences of various factors, including positional parameters of three motors, stiffness coefficient of the springs and frequency ratio on synchronization behaviour, are numerically analysed in the steady state. Additionally, the Runge-Kutta algorithm with adaptive control is employed to build an electromagnetic coupling model, and the relationships between the synchronization state of the system and its mechanical-electrical coupling characteristics are investigated. Finally, an experimental prototype is designed to validate the theory and numerical analysis. The research result shows that the in-phase synchronization of three co-rotating rotors coupled with springs is easy to implement with the selection of a sufficiently large stiffness.

Keywords: synchronization, dynamic characteristic, synchronous conditions, stability criterion, springs

\section{Highlights \\ - A vibrating mechanism of a three co-rotating rotor system coupled with springs is proposed. \\ - $\quad$ The synchronization characteristics of the system are investigated using theory and numerical analysis. \\ - $\quad$ The stable phase difference of three motors are stabilized at zero by the selection of a sufficiently large stiffness. \\ - An experimental prototype is designed to prove the correctness of theory and numerical analysis. \\ - $\quad$ The presented model can be applied to high capacities and efficiencies in the DFSS.}

\section{O INTRODUCTION}

Vibration utilization has always played an important role in a variety of manufacturing industries, such as the vibration conveyer, vibration-impact pile driver, vibratory centrifuge, vibratory crusher, vibratory feeder, etc. The vibrating screen is the most common vibration utilization equipment, especially in petroleum drilling engineering. The vibrating screen is a kind of solid control equipment to separate drilling cuttings from circulating drilling fluid in the process of drilling, which not only undertakes the task of removing a large number of cuttings but can also create a necessary condition for the normal operation of the next solid control equipment. Studies on the drilling fluid shale shaker (DFSS) focus on structural design, screening performance, synchronization theory, etc. In the structural design and screening performance of the vibrating screen, Baragetti suggested increasing the structural and functional performance of the screen by means of a modification of the two side-walls of the mechanical system and studied the dynamic and structural behaviour of the original and modified vibrating screen by using theoretical and numerical models [1]. Dong et al. adopted a three-dimensional discrete element method to study the effect of aperture shape on particle flow and separation in a vibrating screen process [2] and [3]. For the synchronization theory of rotors, Blekhman first proposed a method of the direct separation of motions to solve many engineering problems [4] to [6]. Balthazar et al. investigated the synchronization of two unbalanced rotating motors mounted on the horizontal beam by means of numerical simulations [7] and [8]. Subsequently, Wen et al. [9] and [10] investigated the synchronization problem of two motors in a nonresonance system by using small parameter averaging method, and various synchronous vibrating machines were invented to improve productivity [9] and [10]. Based on Wen's method, Zhang et al. investigated the synchronization of two or three exciters in a farresonant vibrating system of plane motion [11] and [12]. Fang and Hou [13] and Fang et al. [14] discussed the dynamic characteristics of a rotor-pendula system via theoretical analysis and numerical simulations; they determined that the synchronous behaviour 
is determined by mass ratio coefficients, structure parameters, rotating directions, and frequency ratios. Chen et al. used the average method of small parameters to explore the synchronization of two eccentric rotors with a common rotational axis in the far-resonant spatial system. It can be found that the phase difference of two eccentric rotors with a common rotational axis is easily approaching $\pi$ during the running process of the steady-state [15] and [16]. Furthermore, Huang et al. [17] and [18] and Kong et al. [19] and [20] studied the composite synchronization of the vibrating system driven by a multi-motor by applying a cross-coupling control strategy and modified master-slave control structure.

Nowadays, on the one hand, with the rapid development of horizontal drilling technology, the DFSS takes a higher demand for its processing capacity and separating efficiency. On the other hand, as the space of on-site drilling is limited, many companies have proposed improving the processing capacity by increasing the screen layers, which caused the total mass of the vibrating body to increase, and the vibrating system driven by multiple motors are required to achieve a greater exciting force. Hence, many scholars developed a vibrating system with multiple non-identical exciters in a farresonant vibrating system to apply in DFSS [21] to [25]. However, for the synchronization of three nonidentical coupled exciters, those results prove that the phase difference of co-rotating motors stabilized in the neighbourhood of $\pi$ and the exciting force of two exciters are counteracted each other [11]. To improve the amplitude and screening efficiency of the system, a vibrating mechanism of a three co-rotating rotor system coupled with springs in a non-resonance system is proposed for designing large-sized and heavy-duty vibrating screens in petroleum drilling engineering.

In this paper, to further explore the synchronous mechanism of the proposed system and master its synchronous characteristics, the main contents are as follows: In Section 1, a mechanical model of three co-rotating rotors coupled with springs is introduced. Then, the synchronous conditions and the stability criterion of the system are obtained. Next, the influence of positional parameters of three motors, stiffness coefficient of the coupling springs, frequency ratio, the total mass of the system etc., on the steady phase difference are discussed in Section 2. In Section 3, we studied the relationships between synchronization state of the system and its mechanical-electrical coupling characteristics by utilizing the Runge-Kutta algorithm with adaptive control. In addition, an experimental prototype of three co-rotating rotors system coupled with springs is designed and manufactured. Synchronous tests and dynamic tests of the vibrating system are implemented in Section 4. Finally, several important conclusions are summarized in Section 5.

\section{SYNCHRONIZATION MECHANISM}

\subsection{Mechanical Model and Dynamical Equations}

Fig. 1 shows a vibrating system driven by three corotating rotors coupled with springs, which consists of three motors, a rigid frame, an elastic foundation, two coupling springs and four supporting springs. Unbalanced rotors actuated by three identical asynchronous motors are modelled with an eccentric

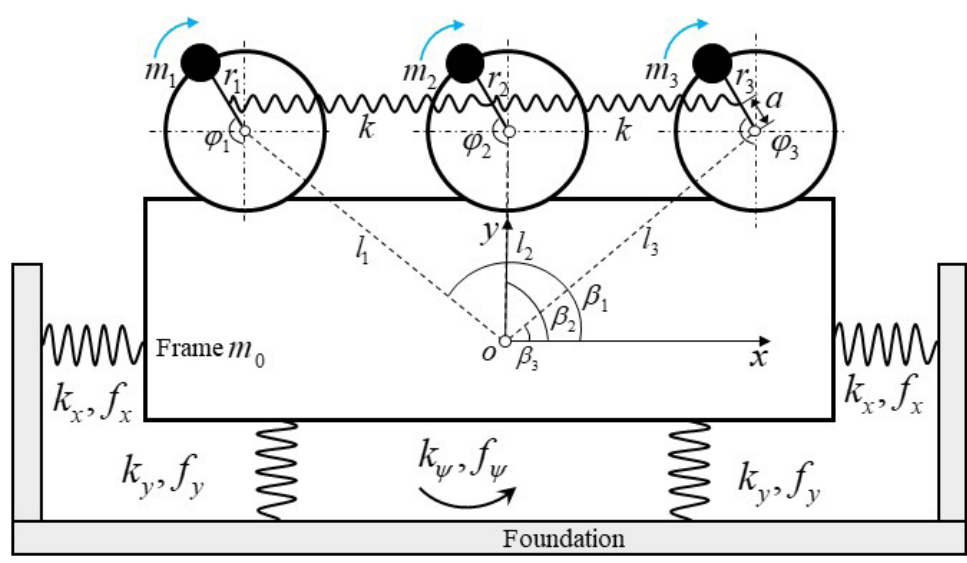

a)

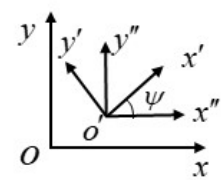

b)

Fig. 1. A vibrating system driven by three co-rotating rotors coupled with springs: a) simplified mechanical model; and b) coordinate system 
lump $m_{i}$ and attached eccentric length $r_{i}(i=1,2,3)$. Three motors are parallelly installed on a rigid frame, and the adjacent two motors are connected with a spring with a stiffness coefficient $k$. The distance from the rotating centre of each motor to the connection of the end of coupling spring is $a$. The vibrating body is connected with a fixed foundation by four supporting springs with stiffness $k_{j}$ and damping $f_{j}$ in $j$-direction. When three motors are simultaneously provided an electromagnetic force, the impact energy produced by its asynchronous motion is absorbed by the coupling springs during the start-up stage of the system. Then the impact energy is absorbed by the coupling springs is increasingly released as the operation of the vibrating system. The steady-state motion of the system is finally implemented under the action of the coupling springs. In the vibrating system, choosing $q=\left[x, y, \psi, \varphi_{1}, \varphi_{2}, \varphi_{3}\right]$ as a generalized coordinate. Then, the generalized active forces of the system are $Q_{j}=\left[0,0,0, T_{e 1}, T_{e 2}, T_{e 3}\right]$ in the $-q_{j}$ direction. Due to the mass of three motors being far less than the rigid frame $\left(m_{i}<<m_{0}\right)$ and the swaying displacement being extremely small $(\psi<<1)$, the coupling inertial moment caused by an asymmetric installation of three motors can be ignored. Consider that the three motors are symmetrically arranged on the rigid frame, and its structure parameters satisfy: $l_{1}=l_{3}=l, l_{2}=l \sin \beta$, $\beta_{1}=\pi-\beta, \beta_{2}=\pi / 2, \beta_{3}=\beta$. According to the general form of Lagrange's equation, the dynamics equations of the vibrating system are derived:

$$
\begin{aligned}
& M \ddot{x}+f_{x} \dot{x}+k_{x} x=\sum_{i=1}^{3} m_{i} r_{i}\left[\ddot{\varphi}_{i} \cos \varphi_{i}-\dot{\varphi}_{i}^{2} \sin \varphi_{i}\right], \\
& M \ddot{y}+f_{y} \dot{y}+k_{y} y=\sum_{i=1}^{3} m_{i} r_{i}\left[-\ddot{\varphi}_{i} \sin \varphi_{i}-\dot{\varphi}_{i}^{2} \cos \varphi_{i}\right], \\
& J \ddot{\psi}+f_{\psi} \dot{\psi}+k_{\psi} \psi=\sum_{i=1}^{3} m_{i} l_{i} r_{i}\left[\ddot{\varphi}_{i} \sin \left(\varphi_{i}+\beta_{i}\right)\right. \\
& \left.\quad+\dot{\varphi}_{i}^{2} \cos \left(\varphi_{i}+\beta_{i}\right)\right], \\
& J_{1} \ddot{\varphi}_{1}+f_{1} \dot{\varphi}_{1}=T_{e 1}+m_{1} r_{1} \ddot{x} \cos \varphi_{1}-m_{1} r_{1} \ddot{y} \sin \varphi_{1} \\
& \quad+m_{1} r_{1} l_{1} \ddot{\psi} \sin \left(\varphi_{1}+\beta_{1}\right)-m_{1} r_{1} l_{1} \dot{\psi}^{2} \cos \left(\varphi_{1}+\beta_{1}\right) \\
& +k a^{2} \sin \left(\varphi_{2}-\varphi_{1}\right)-k l a \cos \left(\varphi_{1}-\beta\right)+k a l \sin \beta \sin \varphi_{1} \\
& +k l \cos \beta f_{1}\left(k, \varphi_{1}, \varphi_{2}, \beta, l, a\right), \\
& J_{2} \ddot{\varphi}_{2}+f_{2} \dot{\varphi_{2}}=T_{e 2}+m_{2} r_{2} \ddot{x} \cos \varphi_{2}-m_{2} r_{2} \ddot{y} \sin \varphi_{2} \\
& \quad+m_{2} r_{2} l_{2} \ddot{\psi} \sin \left(\varphi_{2}+\beta_{2}\right)-m_{2} r_{2} l_{2} \dot{\psi}^{2} \cos \left(\varphi_{2}+\beta_{2}\right) \\
& \quad-k a^{2} \sin \left(\varphi_{2}-\varphi_{1}\right)+k a^{2} \sin \left(\varphi_{3}-\varphi_{2}\right) \\
& \quad+k l \cos \beta f_{2}\left(k, \varphi_{1}, \varphi_{2}, \beta, l, a\right),
\end{aligned}
$$

$$
\begin{aligned}
& J_{3} \ddot{\varphi}_{3}+f_{3} \dot{\varphi}_{3}=T_{e 3}+m_{3} r_{3} \ddot{x} \cos \varphi_{3}-m_{3} r_{3} \ddot{y} \sin \varphi_{3} \\
& \quad+m_{3} r_{3} l_{3} \ddot{\psi} \sin \left(\varphi_{3}+\beta_{3}\right)+k a l \sin \beta \sin \varphi_{3} \\
& \quad-m_{3} r_{3} l_{3} \dot{\psi}^{2} \cos \left(\varphi_{3}+\beta_{3}\right)-k a^{2} \sin \left(\varphi_{3}-\varphi_{2}\right) \\
& \quad+k l a \cos \left(\varphi_{3}+\beta\right)+k l \cos \beta f_{3}\left(k, \varphi_{1}, \varphi_{2}, \beta, l, a\right),
\end{aligned}
$$

where $M=m_{0}+\sum_{1}^{3} m_{i}, \quad J=J_{0}+\sum_{1}^{3} m_{i} l_{i}^{2}+\sum_{1}^{3} m_{i} r_{i}^{2}$, $J_{1}=J_{o 1}+m_{1} r_{1}^{2}, \quad J_{2}=J_{o 2}+m_{2} r_{2}^{2}, \quad J_{3}=J_{o 3}+m_{3} r_{3}^{2}$.

Here $M$ is the total mass of the system; $J$ is the rotational inertia of the system; $f_{i}\left(k, \varphi_{1}, \varphi_{2}, \beta, l, a\right)$ is a coupling term of the springs, and its expressions are given in the Appendix.

\subsection{Steady-State Response}

Due to the motion of the system changing periodically during the running process of the steady-state, the average velocity of three motors is also periodic, and their average values with the least common multiple period $T$ are approximately equal to a constant $\omega_{m}$ :

$$
\omega_{m}=\frac{1}{T} \int_{t_{0}}^{t_{0}+T} \dot{\varphi} d t=\text { constant. }
$$

Assuming the average phase $\varphi$ of the three motors in steady state, and their phase differences are expressed by $\alpha_{12}$ and $\alpha_{23}$, respectively, i.e. $\varphi_{1}-\varphi_{2}=\alpha_{12}$, $\varphi_{2}-\varphi_{3}=\alpha_{23}$. Hence, we have

$$
\varphi_{1}=\varphi+\frac{1}{2} \alpha_{12}, \varphi_{2}=\varphi-\frac{1}{2} \alpha_{12}, \varphi_{3}=\varphi-\frac{1}{2} \alpha_{12}-\alpha_{23} .
$$

Considering the coefficients of the instantaneous change with the average speed of three motors and their phase difference are expressed as $\varsigma_{0}, \varsigma_{12}, \varsigma_{23}$, respectively. i.e.,

$$
\dot{\varphi}=\omega_{m}\left(1+\varsigma_{0}\right), \quad \Delta \dot{\alpha}_{12}=\omega_{m} \varsigma_{12}, \quad \Delta \dot{\alpha}_{23}=\omega_{m} \varsigma_{23} .
$$

Introducing small parameters $\varepsilon_{i}(i=1,2,3)$ to Eq. (3), we know that the vibrating system operates at a steady state when the average values $\varepsilon_{i}(i=1,2,3)$ with one period are equal to zero. Hence, the acceleration of three motors can be written as follows:

$$
\begin{aligned}
& \dot{\varphi}_{1}=\omega_{m}\left(1+\varsigma_{0}+\frac{1}{2} \varsigma_{12}\right)=\left(1+\varepsilon_{1}\right) \omega_{m}, \\
& \dot{\varphi}_{2}=\omega_{m}\left(1+\varsigma_{0}+\frac{1}{2} \varsigma_{12}\right)=\left(1+\varepsilon_{2}\right) \omega_{m}, \\
& \dot{\varphi}_{3}=\omega_{m}\left(1+\varsigma_{0}+\frac{1}{2} \varsigma_{12}-\varsigma_{23}\right)=\left(1+\varepsilon_{3}\right) \omega_{m} .
\end{aligned}
$$

Furthermore, introducing following dimensionless parameters: 
$\varsigma_{x}=\frac{f_{x}}{2 \omega_{n x} M}, \varsigma_{y}=\frac{f_{y}}{2 \omega_{n y} M}, \varsigma_{\psi}=\frac{f_{\psi}}{2 \omega_{n \psi} J}$,

$n_{j}=\frac{\omega_{m}}{\omega_{n j}}, \eta_{1}=\frac{m_{1}}{m_{0}}, \eta_{2}=\frac{m_{2}}{m_{0}}, \eta_{3}=\frac{m_{3}}{m_{0}}, j=x, y, \psi$,

$r_{m}=\frac{m_{0}}{M}, l_{e}=\sqrt{\frac{J}{M}}, r_{l i}=\frac{l_{i}}{l_{e}}, r_{r i}=\frac{r_{i}}{r_{0}}, i=1,2$,

where, $\omega_{n x}=\sqrt{k_{x} / M}, \omega_{n y}=\sqrt{k_{y} / M}, \omega_{n \psi}=\sqrt{k_{\psi} / J}$.

Here, $m_{0}$ is the standard mass of the system and $r_{0}$ is the standard radius of three rotors. Inserting the dimensionless parameters Eq. (6) into Eq. (1) yields the dimensionless formulas of the dynamic equations of the system in $j-(j=\mathrm{x}, \mathrm{y}, \psi)$ direction as:

$$
\begin{aligned}
& \ddot{x}+2 \varsigma_{x} \omega_{n x} \dot{x}+\omega_{n x}{ }^{2} x \\
& =-\omega_{m}{ }^{2} r_{m} r_{0}\left[\begin{array}{l}
\eta_{1} r_{r 1} \sin \varphi_{1}+\eta_{2} r_{r 2} \sin \varphi_{2} \\
+\eta_{3} r_{r 3} \sin \varphi_{3}
\end{array}\right], \\
& \ddot{y}+2 \varsigma_{y} \omega_{n y} \dot{y}+\omega_{n y}{ }^{2} y \\
& =-\omega_{m}{ }^{2} r_{m} r_{0}\left[\begin{array}{l}
\eta_{1} r_{r 1} \cos \varphi_{1}+\eta_{2} r_{r 2} \cos \varphi_{2} \\
+\eta_{3} r_{r 3} \cos \varphi_{3}
\end{array}\right], \\
& \ddot{\psi}+2 \varsigma_{\psi} \omega_{n \psi} \dot{\psi}+\omega_{n \psi}{ }^{2} \psi \\
& =\frac{\omega_{m}{ }^{2} r_{m} r_{0}}{l_{e}}\left[\begin{array}{l}
\eta_{1} r_{l 1} r_{r 1} \cos \left(\varphi_{1}+\beta_{1}\right) \\
+\eta_{2} r_{l 2} r_{r 2} \cos \left(\varphi_{2}+\beta_{2}\right) \\
+\eta_{3} r_{l 3} r_{r 3} \cos \left(\varphi_{3}+\beta_{3}\right)
\end{array}\right] .
\end{aligned}
$$

When the vibrating system operates at the steady state, the periodic solutions of the system in $j-$ $(j=\mathrm{x}, \mathrm{y}, \psi)$ direction can be expressed as:

$$
\begin{aligned}
x= & -r_{m} r_{0} \mu_{x}\left[\eta_{1} r_{r 1} \sin \left(\varphi_{1}-\gamma_{x}\right)\right. \\
& \left.+\eta_{2} r_{r 2} \sin \left(\varphi_{2}-\gamma_{x}\right)+\eta_{3} r_{r 3} \sin \left(\varphi_{3}-\gamma_{x}\right)\right], \\
y= & -r_{m} r_{0} \mu_{y}\left[\eta_{1} r_{r 1} \cos \left(\varphi_{1}-\gamma_{y}\right)\right. \\
& \left.+\eta_{2} r_{r 2} \cos \left(\varphi_{2}-\gamma_{y}\right)+\eta_{3} r_{r 3} \cos \left(\varphi_{3}-\gamma_{y}\right)\right], \\
\psi & =\frac{r_{m} r_{0} \mu_{\psi}}{l_{e}}\left[\eta_{1} r_{l 1} r_{r 1} \cos \left(\varphi_{1}+\beta_{1}-\gamma_{\psi}\right)\right. \\
& +\eta_{2} r_{l 2} r_{r 2} \cos \left(\varphi_{2}+\beta_{2}-\gamma_{\psi}\right) \\
& \left.+\eta_{3} r_{l 3} r_{r 3} \cos \left(\varphi_{3}+\beta_{3}-\gamma_{\psi}\right)\right],
\end{aligned}
$$

here,

$$
\begin{aligned}
& \mu_{j}=\frac{n_{j}^{2}}{\sqrt{\left(1-n_{j}^{2}\right)^{2}+\left(2 \varsigma_{j} n_{j}\right)^{2}}}, \\
& \gamma_{j}=\left\{\begin{array}{ll}
\arctan \left(\frac{2 \varsigma_{j} n_{j}}{1-n_{j}^{2}}\right) & 1-n_{\psi}{ }^{2} \geq 0 \\
\pi+\arctan \left(\frac{2 \varsigma_{j} n_{j}}{1-n_{j}^{2}}\right) & \text { other }
\end{array} .\right.
\end{aligned}
$$

\subsection{Synchronous Conditions}

Since the far-resonant vibrating system is commonly used in engineering applications, the exciting frequency of the system is 3 to 10 times greater than its natural frequency, i.e. $n_{j} \geq 3$, and the vibrating system with small damping $\left(\varsigma_{j} \leq 0.07\right)$ [10]. Hence, $\mu_{j} \cong n_{j}^{2} /\left(n_{j}^{2}-1\right), \quad \gamma_{j}=\pi+\arctan \left(2 \varsigma_{j} n_{j} /\left(1-n_{j}^{2}\right)\right)$. In light of literature [11] and [12], the rated slip of the motors ranges from 0.02 to 0.08 during the running process of the steady-state. When the three rotors are rotated with an equal velocity $\left(\dot{\varphi}_{i}=\omega_{m}\right)$, their electromagnetic torque can be written in the form:

$$
T_{e i}=T_{e 0 i}-\bar{k}_{e 0 i} \bar{\varepsilon}_{i},
$$

where $T_{e 0 i}$ and $\bar{k}_{e 0 i}$ are given in the literature [14]. Differentiating Eq. (8) to obtain $\dot{x}, \ddot{x}, \dot{y}, \ddot{y}, \dot{\psi}$ and $\ddot{\psi}$. Then, inserting them into the dynamical equations of the rotors in Eq. (1), and integrating them with one period, we obtain the matrix form of $\bar{\varepsilon}_{i}$ in the form:

$$
\mathbf{P} \dot{\bar{\varepsilon}}=\mathbf{Q} \bar{\varepsilon}+\mu,
$$

where $\bar{\varepsilon}=\left[\begin{array}{lll}\bar{\varepsilon}_{1} & \bar{\varepsilon}_{2} & \bar{\varepsilon}_{3}\end{array}\right]^{\mathrm{T}}, \boldsymbol{\mu}=\left[\begin{array}{lll}\mu_{1} & \mu_{2} & \mu_{3}\end{array}\right]^{\mathrm{T}}$,

$$
\mathbf{P}=\left[\begin{array}{ccc}
\rho_{11} & \chi_{12}^{\prime} & \chi_{13}^{\prime} \\
\chi_{21}^{\prime} & \rho_{12} & \chi_{23}^{\prime} \\
\chi_{31}^{\prime} & \chi_{32}^{\prime} & \rho_{13}
\end{array}\right], \quad Q=-\omega_{m}\left[\begin{array}{ccc}
k_{11} & \chi_{12} & \chi_{13} \\
\chi_{21} & k_{22} & \chi_{23} \\
\chi_{31} & \chi_{32} & k_{33}
\end{array}\right] .
$$

Parameters $\rho_{11}, \chi_{12}^{\prime}$, etc. are shown in the Appendix.

The symbol $\mathbf{P}$ represents the coupling matrix of three rotors; the symbol $\mathbf{Q}$ is defined as the stiffness matrix of the vibrating system; the symbol $\boldsymbol{\mu}$ is the torque coupling matrix of three rotors. When the vibrating system operates at the steady state, the coefficients of the instantaneous change are approximate to zero, i.e., $\varsigma_{0}=0, \varsigma_{12}=0, \varsigma_{23}=0$. Hence, the values of $\bar{\varepsilon}$ also tend to zero. Inserting them into Eq. (10), we obtain $\boldsymbol{\mu}=0$, and adding them together to obtain the following expression:

$$
\begin{aligned}
& \sum_{i=1}^{3} T_{e 0 i}-\omega_{m} \sum_{i=1}^{3} f_{i} \\
& -\frac{1}{2} m_{0} r_{0}^{2} \omega_{m}{ }^{2} \sum_{i=1}^{3}\left(\eta_{i}{ }^{2} r_{r i}{ }^{2} W_{s i}\right)+k l \cos \beta F\left(\alpha_{12}, \alpha_{23}\right) / 2 \pi \\
& +\frac{1}{2} m_{0} r_{0}^{2} \omega_{m}{ }^{2} \eta_{1} \eta_{3} r_{r 1} r_{r 3} \\
& \cdot\left[-W_{s 13} \sin \left(\alpha_{12}+\alpha_{23}+\theta_{s 13}\right)-W_{s 13} \cos \left(\alpha_{12}+\alpha_{23}+\theta_{s 13}\right)\right] \\
& -m_{0} r_{0}{ }^{2} \omega_{m}{ }^{2} \eta_{2} \eta_{3} r_{r 2} r_{r 3} W_{s 23} \cos \left(\alpha_{23}+\theta_{s 23}\right) \\
& -m_{0} r_{0}{ }^{2} \omega_{m}{ }^{2} \eta_{1} \eta_{2} r_{r 1} r_{r 2} W_{s 12} \cos \left(\alpha_{12}+\theta_{s 12}\right)=0,
\end{aligned}
$$

where 


$$
\begin{aligned}
& F\left(\alpha_{12}, \alpha_{23}\right)=\int_{0}^{2 \pi} \frac{-2 l a \cos \beta \sin \frac{\alpha_{12}}{2} \sin \varphi}{\left[\begin{array}{l}
l^{2} \cos ^{2} \beta+2 a^{2}-2 a^{2} \cos \alpha_{12} \\
+4 l a \cos \beta \sin \frac{\alpha_{12}}{2} \cos \varphi
\end{array}\right]^{0.5}} d \varphi
\end{aligned}
$$

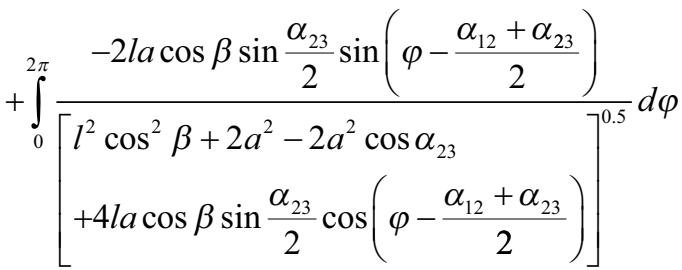

Eq. (11) is the equilibrium equation of the dynamic moment of the whole system. The first term $\left(\sum_{i=1}^{3} T_{e 0 i}\right)$ represents the sum of the output torque of three motors; the second term $\left(\omega_{m} \sum_{i=1}^{3} f_{i}\right)$ is the sum of resistance torque of three motors during operation; the remaining terms denote the mechanical load of three motors operating in the steady state and the coupling torque of those connecting springs among three rotors. Moreover, it can be seen that there is no coupling term with connecting spring in Eq. (11). An optimal zero phase synchronization of three motors is achieved, i.e., $\alpha_{12}=0, \alpha_{23}=0$. That is to say, the deformation rate of these connecting springs is always equal to zero during the running process of the steadystate. Due to $\boldsymbol{\mu}=0$, the difference equations of two of motors can be obtained:

$$
\begin{aligned}
& {\left[T_{e 01}-\omega_{m} f\right]-\left[T_{e 02}-\omega_{m} f_{2}\right]+k a^{2}\left[\sin \alpha_{23}-2 \sin \alpha_{12}\right]} \\
& +\frac{k l \cos \beta}{2 \pi}\left[F_{1}(\varphi)-F_{2}(\varphi)\right] \\
& +\frac{1}{2} m_{0} r_{0}^{2} \omega_{m}{ }^{2}\left[\begin{array}{l}
-\eta_{1}{ }^{2} r_{r 1}{ }^{2} W_{s 1}+\eta_{2}{ }^{2} r_{r 2}{ }^{2} W_{s 2} \\
-2 \eta_{1} \eta_{2} r_{r 1} r_{r 2} W_{c 12} \sin \left(\alpha_{12}+\theta_{c 12}\right)
\end{array}\right] \\
& -\frac{1}{2} m_{0} r_{0}{ }^{2} \omega_{m}{ }^{2} \eta_{1} \eta_{3} r_{r 1} r_{r 3}\left[\begin{array}{l}
W_{c 13} \sin \left(\alpha_{12}+\alpha_{23}+\theta_{c 13}\right) \\
+W_{s 13} \sin \left(\alpha_{12}+\alpha_{23}+\theta_{s 13}\right)
\end{array}\right] \\
& +\frac{1}{2} m_{0} r_{0}{ }^{2} \omega_{m}{ }^{2} \eta_{2} \eta_{3} r_{r 2} r_{r 3}\left[\begin{array}{l}
W_{c 23} \sin \left(\alpha_{23}+\theta_{c 23}\right) \\
+W_{s 23} \cos \left(\alpha_{23}+\theta_{s 23}\right)
\end{array}\right]=0 \\
& {\left[T_{e 02}-\omega_{m} f_{2}\right]-\left[T_{e 03}-\omega_{m} f_{3}\right]} \\
& +k a^{2}\left[\sin _{12}-2 \sin _{23}\right]+\frac{k l \cos \beta}{2 \pi}\left[F_{2}(\varphi)-F_{3}(\varphi)\right] \\
& +\frac{1}{2} m_{0} r_{0}{ }^{2} \omega_{m}{ }^{2} \eta_{1} \eta_{2} r_{r 1} r_{r 2}\left[\begin{array}{l}
W_{c 12} \sin \left(\alpha_{12}+\theta_{c 12}\right) \\
-W_{s 12} \cos \left(\alpha_{12}+\theta_{s 12}\right)
\end{array}\right] \\
& +\frac{1}{2} m_{0} r_{0}{ }^{2} \omega_{m}{ }^{2}\left[\begin{array}{l}
-\eta_{2}{ }^{2} r_{r 2}{ }^{2} W_{s 2}+\eta_{3}{ }^{2} r_{r 3}{ }^{2} W_{s 3} \\
-2 \eta_{2} \eta_{3} r_{r 2} r_{r 3} W_{c 23} \sin \left(\alpha_{23}+\theta_{c 23}\right)
\end{array}\right] \\
& -\frac{1}{2} m_{0} r_{0}{ }^{2} \omega_{m}{ }^{2} \eta_{1} \eta_{3} r_{r 1} r_{r 3}\left[\begin{array}{l}
W_{c 13} \sin \left(\alpha_{12}+\alpha_{23}+\theta_{c 13}\right) \\
-W_{s 13} \cos \left(\alpha_{12}+\alpha_{23}+\theta_{s 13}\right)
\end{array}\right]=0[(13)
\end{aligned}
$$

Eqs. (12) and (13) are dimensionless difference equations with respect to $\alpha_{12}$ and $\alpha_{23}$, which reveals the coupling property of the system when the vibrating system operates at the steady state.

\subsection{Stability Criterion}

In this study, neglecting the effect of system damping, the vibrating system is encountering not only gravitational forces but also the output torque of motors during the running process of the steadystate. Thereby, three co-rotating rotors system coupled with springs is a nonholonomic conservation system. According to Hamilton's principle, we obtain the following expression:

$$
\int_{0}^{2 \pi}\left[\delta(T-V)+\sum_{i=1}^{3} Q_{i} \delta q_{i}\right] d \varphi=0
$$

where $T, V, Q_{i}$ and $q_{i}$ represent the total kinetic energy, the total potential energy, the generalized force, and the generalized coordinate of the system, respectively. From the model proposed in Fig. 1, we obtain the total kinetic energy of the system:

$$
T=\frac{1}{2} m_{0}\left(\dot{x}^{2}+\dot{y}^{2}\right)+\frac{1}{2} J_{0} \dot{\psi}^{2}+T_{z} .
$$

Here $T_{z}$ is the sum of kinetic energy with three motors. Since their rotation speeds are identical to each other during the running process of the steadystate, $T_{z}$ can be regarded as a constant. The total potential energy of the vibrating system can be written as:

$$
V=\frac{1}{2} k_{x} x^{2}+\frac{1}{2} k_{y} y^{2}+\frac{1}{2} k_{\psi} \psi^{2}+\frac{1}{2} k \Delta_{1}^{2}+\frac{1}{2} k \Delta_{2}^{2} .
$$

The Hamiltonian interaction of the system over one period can be written the following:

$$
H=\int_{0}^{T}(T-V) d t=\int_{0}^{2 \pi}(T-V) d \varphi .
$$

As the model of three co-rotating rotors system has two degrees of freedoms (DOFs), we choose $\Delta \alpha_{12}$, $\Delta \alpha_{23}$ to be a generalized coordinate. Three rotors are rotating with an equal velocity $\left(\omega_{m}\right)$ when the vibrating system operates at the steady state. Simultaneously, the values of $\Delta \alpha_{12}$ and $\Delta \alpha_{23}$ are approximately equal to a constant $\left(\Delta \alpha_{12}{ }^{*}\right.$ and $\left.\Delta \alpha_{23}{ }^{*}\right)$. According to a mechanical system with integrity constraint, the system can be changed from one position to another under the action of conservative forces, and the movement of the system tends to be stable when its Hamiltonian interaction has a minimum. Therefore, a stability criterion of the system can be obtained in the form: 
$\frac{\partial^{2} H}{\partial \Delta \alpha_{12}^{2}}>0, \quad \frac{\partial^{2} H}{\partial \Delta \alpha_{12}^{2}} \cdot \frac{\partial^{2} H}{\partial \Delta \alpha_{23}^{2}}-\left(\frac{\partial^{2} H}{\partial \Delta \alpha_{12} \partial \Delta \alpha_{23}}\right)>0$.

\section{NUMERICAL DISCUSSION}

Some theoretical results with regard to synchronous conditions and stability criteria for the three corotating rotors system coupled with springs are described in the preceding section. From Eqs. (12), (13) and (18), it can be seen that the synchronous state of the system is mainly determined by the positional parameters of three motors, the stiffness coefficient of the coupling springs, the frequency ratio, the total mass of the system, etc. To deeply grasp the influence of various factors on the synchronous state of the system, some numerical analyses for solving Eqs. (12) and (13) under the conditions of Eq. (18) are performed to analyse the influence of positional parameters of the three motors and the frequency ratio on the synchronous characteristic of the vibrating system.

When the vibrating system operates at the steady state, the synchronization state of the system is defined as follows: the phase difference of rotors is always close to $(-\pi / 2, \pi / 2)$ or $\left(-90^{\circ}, 90^{\circ}\right)$, the vibrating system is called the in-phase synchronization. Also, the phase difference of rotors is always close to $(\pi / 2,3 \pi / 2)$ or $\left(90^{\circ}, 270^{\circ}\right)$; the system is called the anti-phase synchronization. Considering installation angle $\beta$ of motors are set as $15^{\circ}, 30^{\circ}, 42^{\circ}, 60^{\circ}$, respectively, and the influence of stiffness coefficient

Table 1. The structural parameters of the vibrating system in engineering

\begin{tabular}{cccc}
\hline unbalanced rotors $(i=1,2,3)$ & a rigid frame & motor & coupling springs \\
\hline & $M=90 \mathrm{~kg}$ & & \\
& $J=6.8 \mathrm{~kg} \cdot \mathrm{m}^{2}$ & & \\
$m_{i}=2 \mathrm{~kg}$ & $k_{x}=8 \times 10^{4} \mathrm{~N} / \mathrm{m}, 6.6 \times 10^{6} \mathrm{~N} / \mathrm{m}$ & $l_{1}=0.35 \mathrm{~m}, 0.52 \mathrm{~m}, 0.64 \mathrm{~m}, 0.85 \mathrm{~m}$ & \\
$r=0.04 \mathrm{~m}$ & $k_{y}=8 \times 10^{4} \mathrm{~N} / \mathrm{m}, 6.6 \times 10^{6} \mathrm{~N} / \mathrm{m}$ & $l_{2}=0.12 \mathrm{~m}, 0.18 \mathrm{~m}, 0.22 \mathrm{~m}, 0.30 \mathrm{~m}$ & $k=1 \sim 4.2 \times 104 \mathrm{~N} / \mathrm{m}$ \\
$\omega_{m}=157 \mathrm{rad} / \mathrm{s}$ & $k_{\psi}=6 \times 10^{3} \mathrm{~N} / \mathrm{m}, 4.96 \times 10^{5} \mathrm{~N} / \mathrm{m}$ & $l_{3}=0.35 \mathrm{~m}, 0.52 \mathrm{~m}, 0.64 \mathrm{~m}, 0.85 \mathrm{~m}$ & $a=0.02 \mathrm{~m}$ \\
$f_{i}=0.02 \mathrm{~N} \cdot \mathrm{s} / \mathrm{m}$ & $f_{x}=1000 \mathrm{~N} \cdot \mathrm{s} / \mathrm{m}$ & $\beta_{1}=160^{\circ}, 78^{\circ}, 20^{\circ}$ & \\
& $f_{y}=1000 \mathrm{~N} \cdot \mathrm{s} / \mathrm{m}$ & & \\
& $f_{\psi}=1000 \mathrm{~N} \cdot \mathrm{s} / \mathrm{m}$ & & \\
& & & \\
\end{tabular}
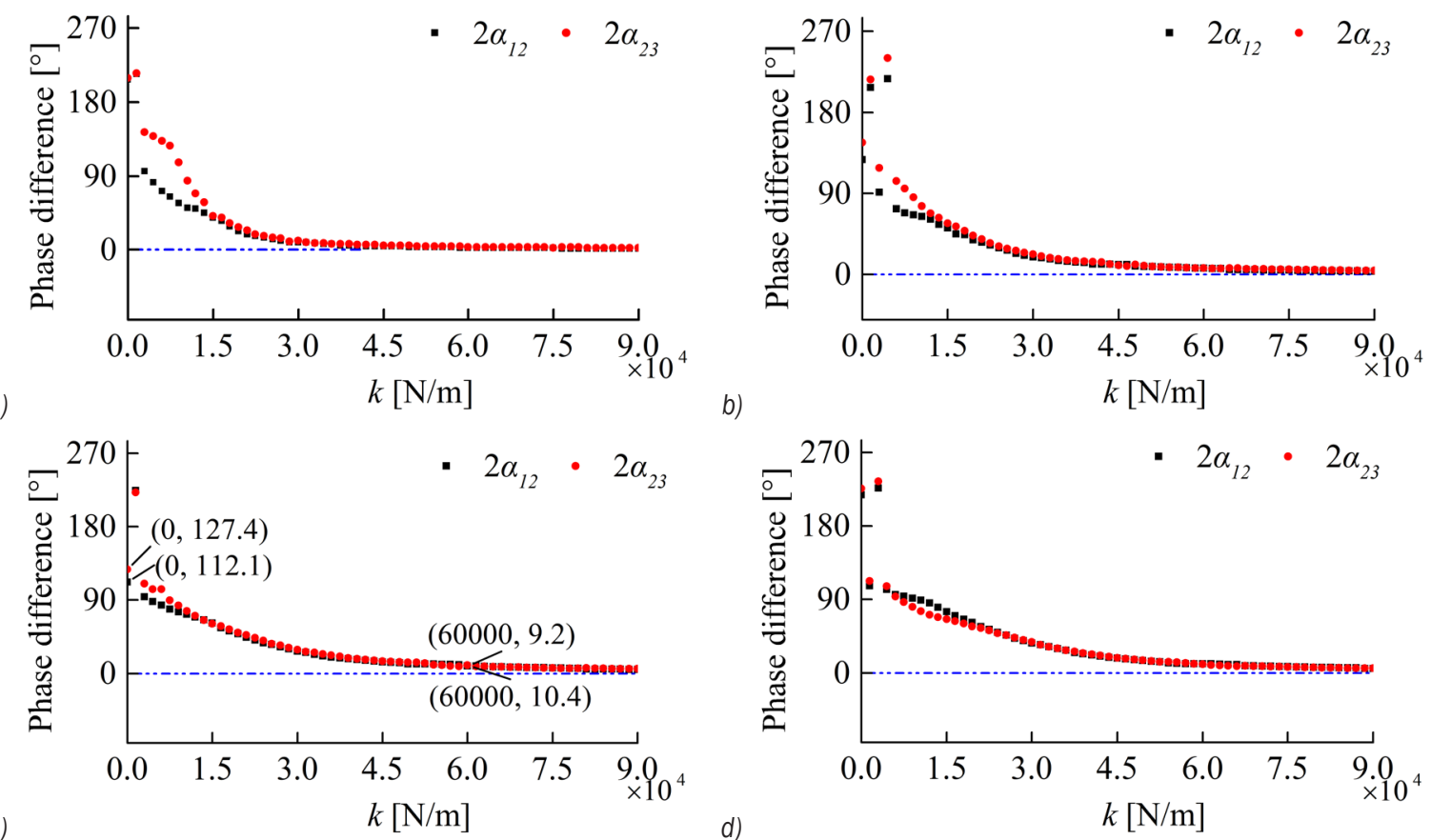

Fig. 2. Installation angle of motors and stiffness coefficient of the coupling springs are major influences on the dynamic characteristics; a) $\beta=15^{\circ}$, b) $\beta=30^{\circ}$, c) $\beta=42^{\circ}$, and d) $\beta=60^{\circ}$ 
of the coupling springs on the synchronization state is presented in Fig. 2. When $k=0$, meaning that there is no coupling springs among these rotors, the phase differences between $2 \alpha_{12}$ and $2 \alpha_{23}$ consistently tend towards anti-phase synchronization. From Fig. 2c, it can be found that the values of $2 \alpha_{12}$ and $2 \alpha_{23}$ are equal to $127.4^{\circ}$ and $112.1^{\circ}$, respectively, when $\beta=42^{\circ}$. For the coupling springs with a small coefficient,
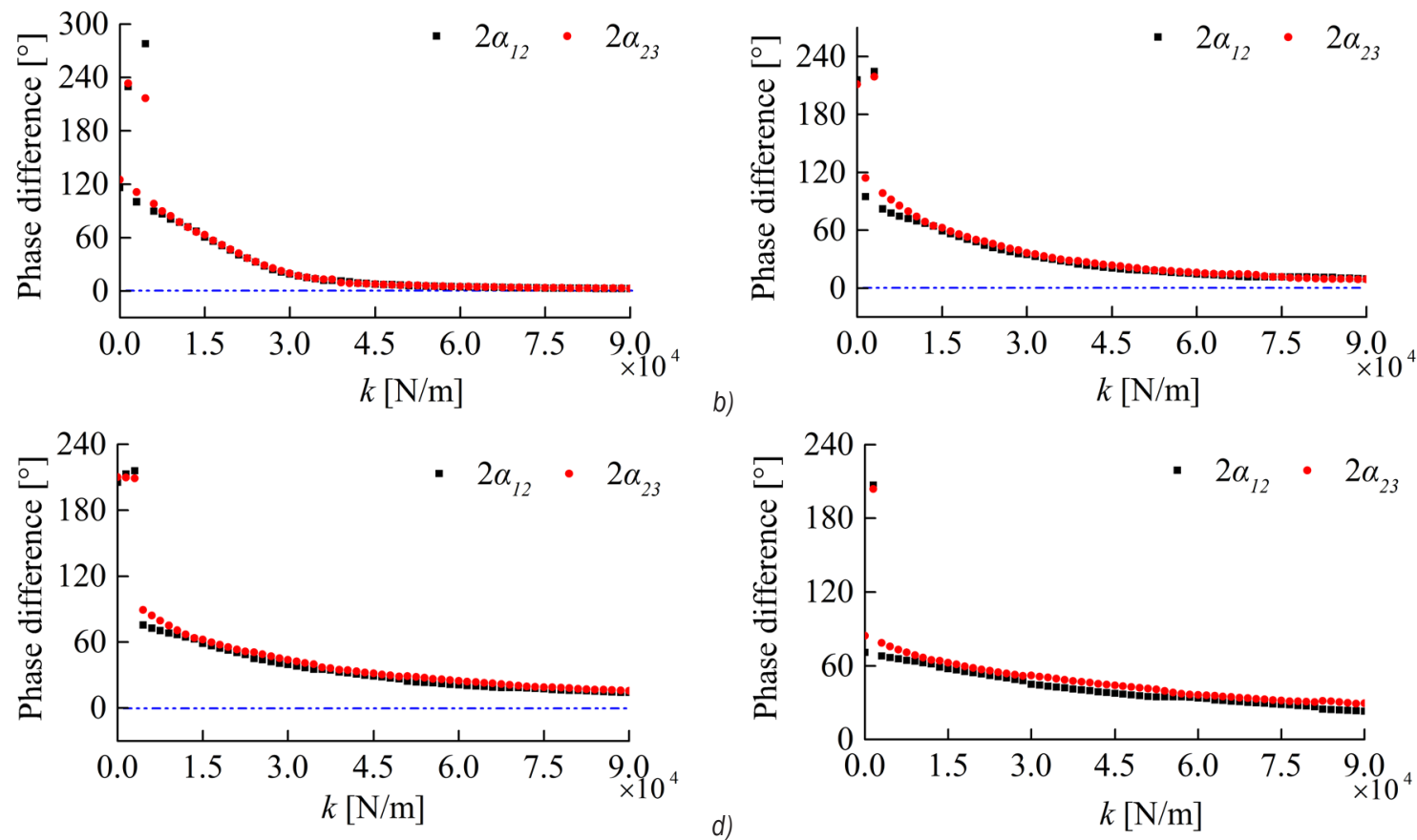

Fig. 3. Installation distance of motors and stiffness coefficient of the coupling springs are major influences on the dynamic characteristics; a) $l=0.32 \mathrm{~m}, b) l=0.64 \mathrm{~m}, \mathrm{c}) l=0.85 \mathrm{~m}$, and d) $l=1.28 \mathrm{~m}$
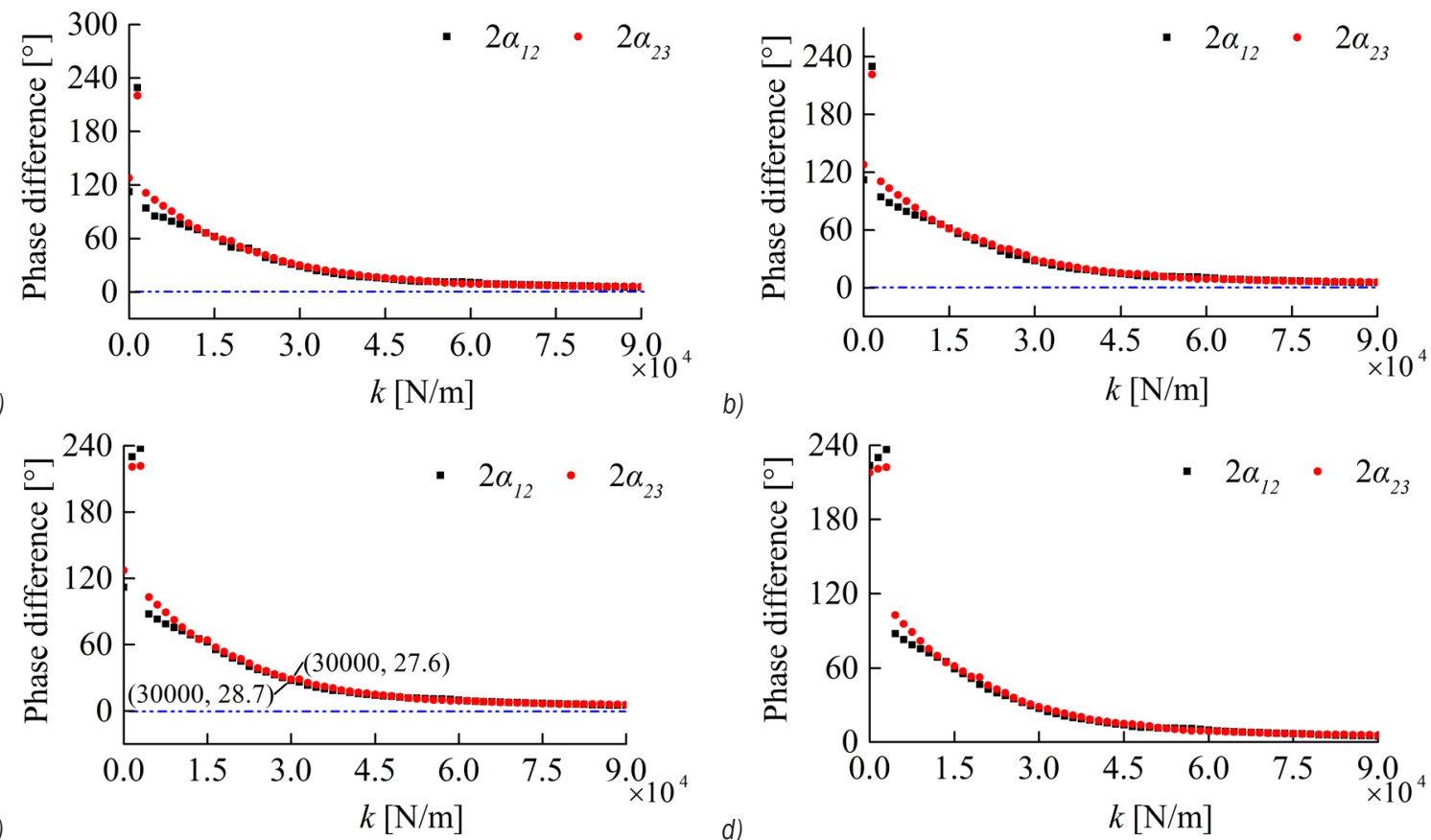

Fig. 4. Frequency ratio and stiffness coefficient of the coupling springs are major influences on the dynamic characteristics; a) $n_{j}=4.47$, b) " $n_{j}=5$, c) $n_{j}=6.8$, and d) $n_{j}=8.95$ 
their elastic force has no influence on synchronous characteristic of the vibrating system. However, with the increasing of $k$ over a critical value, the phase differences between each pair of the rotors gradually stabilize at zero. Accordingly, the synchronous state of the system is suddenly changed from anti-phase synchronization to the in-phase synchronization. Moreover, from the contrasting results shown in Fig. 2 , it is also demonstrated that the installation angles of the three motors have significantly impacted the synchronous behaviour of the vibrating system.

We assume that $\beta=42^{\circ}$ and the value of $l$ is equal to $0.32 \mathrm{~m}, 0.64 \mathrm{~m}, 0.85 \mathrm{~m}, 1.28 \mathrm{~m}$, respectively. The influences of the stiffness coefficient of the coupling springs on synchronization state are illustrated in Fig. 3. Compared with numerical results with different installation distances, the parameter $l$ has an appreciable effect on the synchronous behaviour of the vibrating system whenk $k=0$. In addition, antiphase synchronization occurs for the coupling springs with a small coefficient. However, with the increasing $k$ over a critical value, the phase differences between each pair of the rotors gradually stabilize at zero. Accordingly, it can be concluded that the changing trends closing to zero are different when three motors are installed in different locations.

Fig. 4 shows that the stiffness coefficient of the coupling springs is a major influence on the dynamic characteristics of the vibrating system under the conditions of different frequency ratios. It can be concluded that the frequency ratio of the system has no influence on synchronous behaviour. However, for different frequency ratios, the changing rule of phase difference with changing of stiffness coefficient of the coupling springs in the steady state are consistent with those preceding conclusions in Figs. 2 and 3.

\section{SIMULATION VERIFICATION}

Based on the dynamics Eq. (1), a simulation model with three co-rotating rotors system coupled with springs is established by means of the Runge-Kutta algorithm with adaptive control. The relationships between the synchronization state of the system and their mechanical-electrical coupling characteristics are investigated, and further analysis results are employed to verify the correctness of theoretical derivation and numerical analysis. Simulation parameters are identical with numerical results in Table 1.
3.1 Dynamic Characteristics for $k=0 \mathrm{~N} / \mathrm{m}, l=0.48 \mathrm{~m}$, and $n_{j}=5.48$

Simulation results for $n_{j}(j=x, y, \psi)=5.48, l=0.48 \mathrm{~m}$, $k=0 \mathrm{~N} / \mathrm{m}$ are shown in Fig. 5 . Here, $k_{x}=8 \times 10^{4} \mathrm{~N} / \mathrm{m}$, $k_{y}=8 \times 10^{4} \mathrm{~N} / \mathrm{m}, k_{\psi}=1.28 \times 10^{4} \mathrm{rad} / \mathrm{m}$. The vibrating system is gradually changed from a desynchronynous state to synchronization for about 3 seconds, and the driving torques of three motors are changed near 3.9 $\mathrm{N} \cdot \mathrm{m}, 3.71 \mathrm{~N} \cdot \mathrm{m}, 3.71 \mathrm{~N} \cdot \mathrm{m}$, respectively, as shown in Fig. 5(a). Moreover, three rotors are rotated with the same velocity $152.7 \mathrm{rad} / \mathrm{s}$ when the vibrating system operates at the steady state, the phase difference $2 \alpha_{12}$ between rotor 1 and rotor 2 is stabilized at $-4.49 \mathrm{rad}$ $\left(102.7^{\circ} \triangleq-4.49 \mathrm{rad}-2 \pi\right)$, and the phase difference $2 \alpha_{23}$ between rotor 2 and rotor 3 is stabilized at 8.42 $\operatorname{rad}\left(122.4^{\circ} \triangleq 8.42 \mathrm{rad}-2 \pi\right)$, as shown in Fig. 5f. Compared with numerical result of the corresponding parameter in Fig. 2c, the results show that simulation results are proven to be in good agreement with the numerical results. Figs. 5c, d and e show phase diagrams of the vibrating system in the DOFs. As seen from those diagrams, the rigid frame was not rapidly excited owing to its large mass during the initial process of the vibrating system, which caused the phase diagram of the mass centre of the system to be chaotic in the DOFs. The synchronous behaviour of the vibrating system is gradually implemented as the system kept running, the phase diagram of the mass centre of the system in the $x-y$ plane is a closed ellipse, and its amplitude in the DOFs is $2.82 \times 10^{-3} \mathrm{~m}, 2.8 \times$ $10^{-4} \mathrm{~m}, 3.34 \times 10^{-4} \mathrm{rad}$, respectively, as schematically illustrated in Fig. $5 \mathrm{~g}$.

3.2 Dynamic characteristics for $k=60000 \mathrm{~N} / \mathrm{m}, l=0.48$ $\mathrm{m}$, and $n_{j}=5.48$

For the system parameters in Section 4.1, changing the value $k=60000 \mathrm{~N} / \mathrm{m}$, and simulation results are shown in Fig. 6. When three motors are simultaneously provided with electromagnetic force, the synchronization phenomenon occurs after 4 seconds, and the rotational velocities of three motors are stabilized at $151.8 \mathrm{rad} / \mathrm{s}$, as shown in Fig. 6b. As illustrated in Fig. 6a, the driving torque of three motors in synchronous state are $4.72 \mathrm{~N} . \mathrm{m}, 4.4 \mathrm{~N} \cdot \mathrm{m}$, $4.4 \mathrm{~N} \cdot \mathrm{m}$, respectively. Figs. $6 \mathrm{c}, \mathrm{d}$ and $6 \mathrm{e}$ show phase diagrams of the vibrating system in $x$-, $y$-, and $\psi$ directions, respectively. The results show that the phase diagram of the mass centre of the system is chaotic during the initial process of the vibrating system. Moreover, its phase diagram in the $x-y$ plane is a closed ellipse when the vibrating system operates 

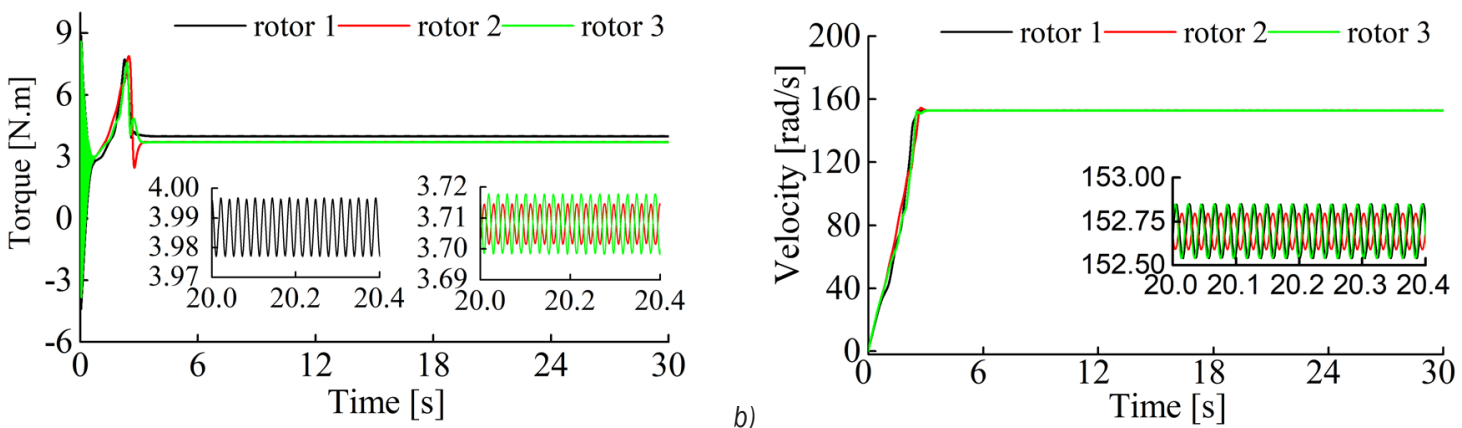

a) Time [s]

b)

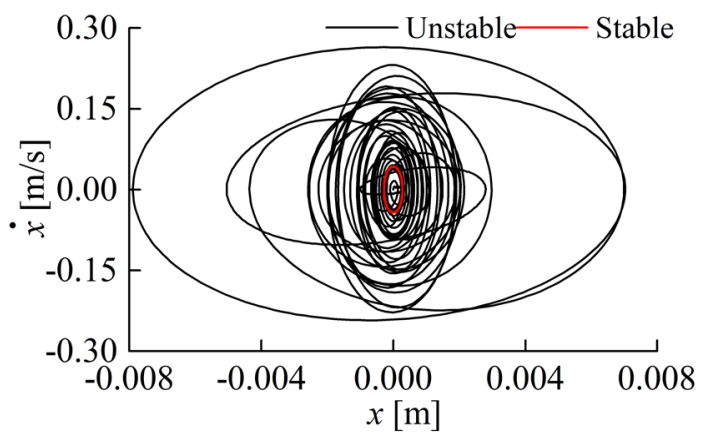

c)
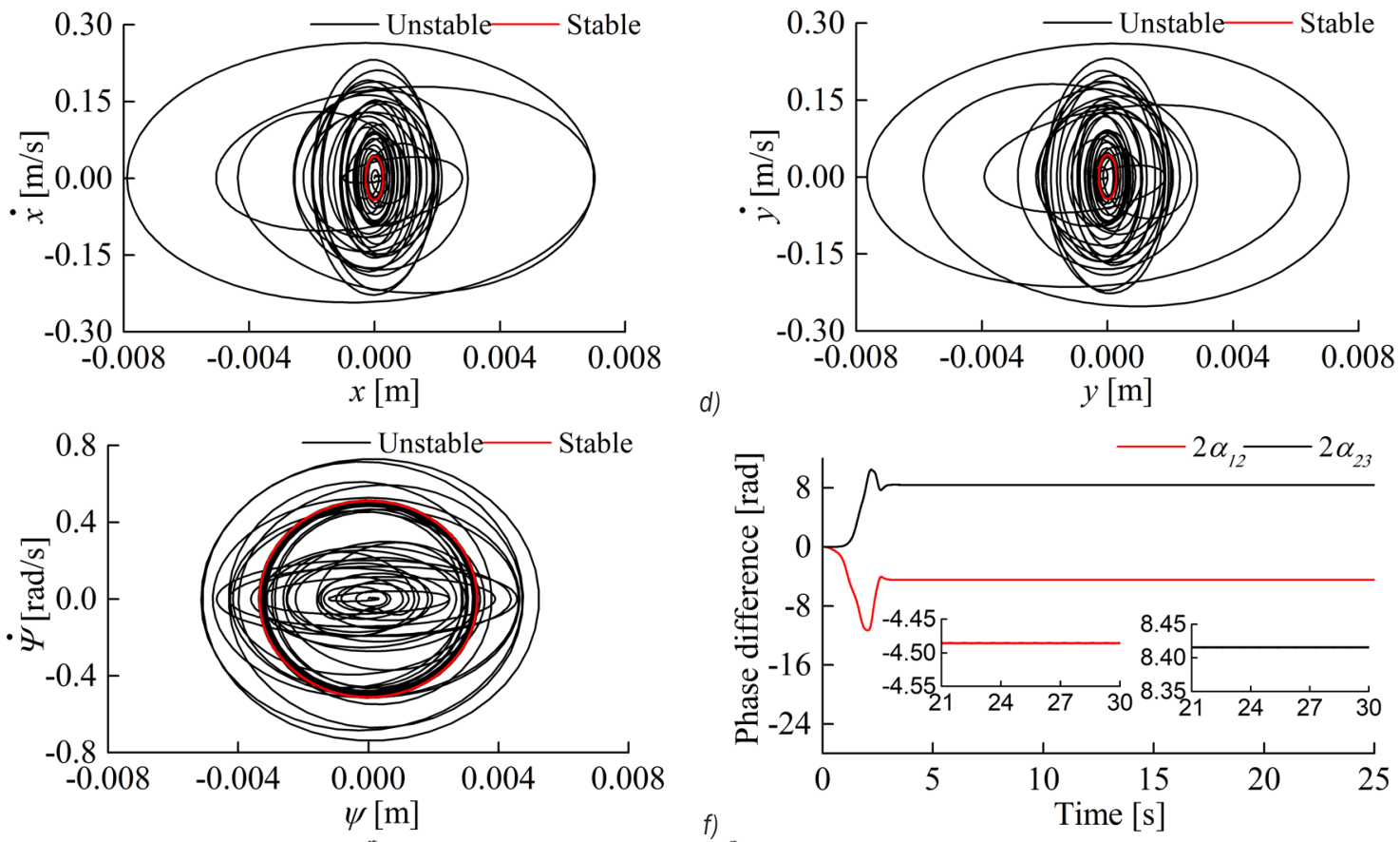

d)

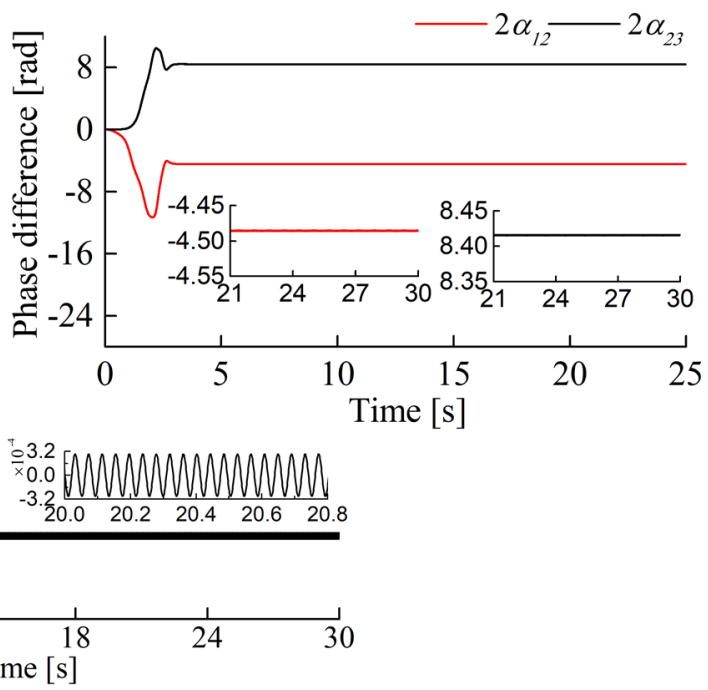

Fig. 5. Simulation results for $k=0 \mathrm{~N} / \mathrm{m}, l=0.48 \mathrm{~m}, n_{j}=5.48$; a) driving torque of three motors, b) rotational velocity of three motors, c) phase diagram of the system in $x$-direction, d) phase diagram of the system in $y$-direction, e) phase diagram of the system in $\psi$-direction, f) phase difference of three motors, and g) displacement response of the rigid frame in $x-, y$-, $\psi$-directions, respectively

at the steady state. The value of $2 \alpha_{12}$ is approximately equal to $0.194 \mathrm{rad}\left(11.1^{\circ} \triangleq 0.194 \mathrm{rad}\right)$, and the value of $2 \alpha_{23}$ is approximately equal to $0.181 \mathrm{rad}\left(10.4^{\circ} \triangleq\right.$ $0.181 \mathrm{rad})$. By comparison, the simulation results are in good agreement with numerical results. Fig. $6 \mathrm{~g}$ shows the amplitude of the mass centre of the system in a synchronous state, and its magnitudes are $3.1 \times$ $10^{-3} \mathrm{~m}, 3.1 \times 10^{-3} \mathrm{~m}, 4.64 \times 10^{-3} \mathrm{rad}$, respectively, as shown in Table 2 . In addition, comparing simulation results in Figs. 5 and 6 , it is demonstrated that synchronous state of the system is significantly changed by those coupling springs among the rotors, which makes the system transit from anti-phase synchronization to the in-phase synchronization. Moreover, it can be seen that adjusting the value of the coupling spring stiffness can make the phase difference close to zero to meet the requirements of the strongly exciting designing large-sized and heavyduty vibrating screens in engineering. 


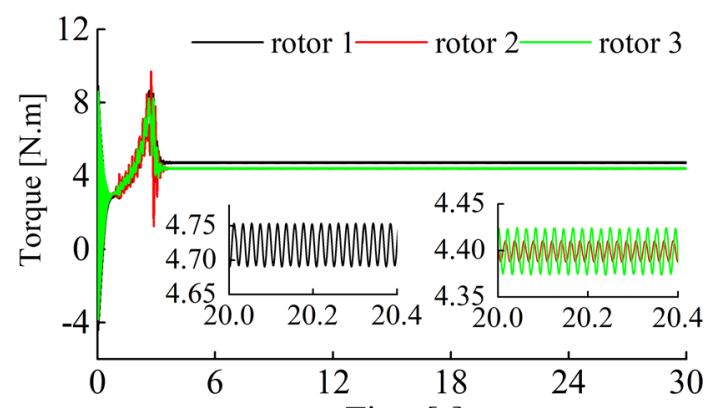

a)
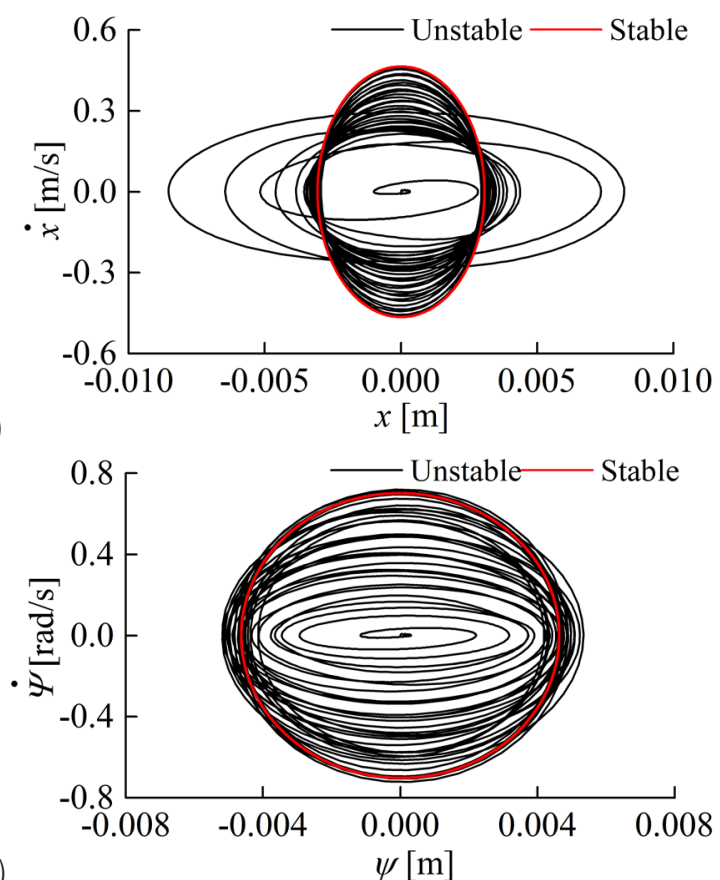

g)

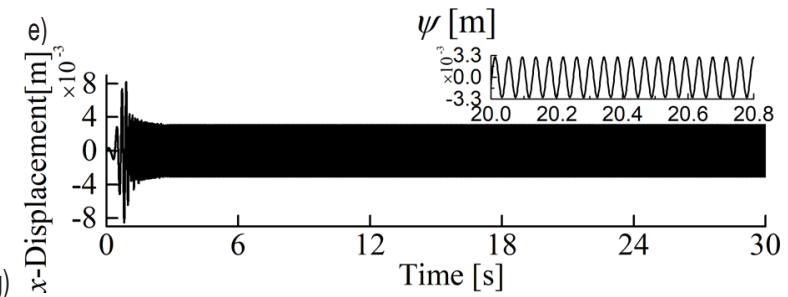

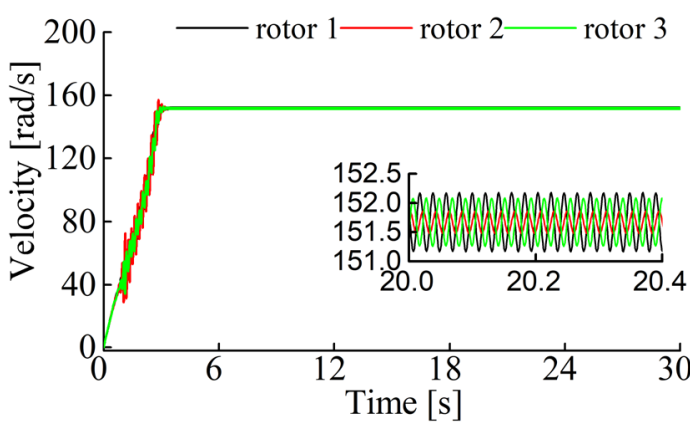
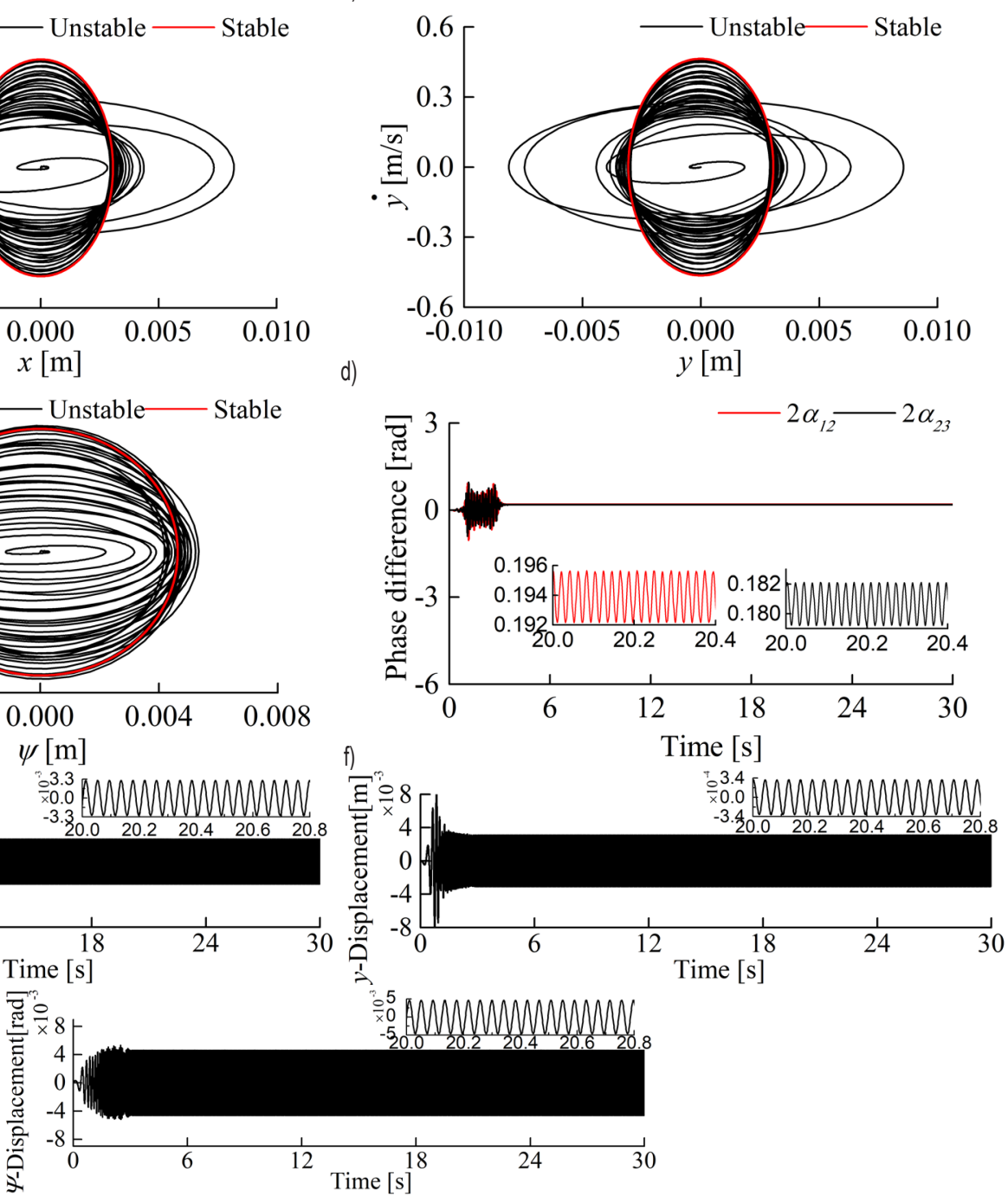

Fig. 6. Simulation results for $k=60000 \mathrm{~N} / \mathrm{m}, l=0.48 \mathrm{~m}, n_{j}=5.48$; a) driving torque of three motors, $b$ ) rotational velocity of three motors, c) phase diagram of the system in $x$-direction, d) phase diagram of the system in $y$-direction, e) phase diagram of the system in $\psi$-direction, f) phase difference of three motors, and g) displacement response of the rigid frame in $x$-, $y$-, $\psi$-directions, respectively

Table 2. Displacement amplitude of the vibrating system with the changing of the stiffness of the coupling springs

\begin{tabular}{lccc}
\hline & $x$-direction $[\mathrm{mm}]$ & $y$-direction $[\mathrm{mm}]$ & $\psi$-direction $[\mathrm{mm}]$ \\
\hline$k=0$ & 2.82 & 0.28 & 3.34 \\
\hline$k=60000$ & 3.1 & 3.1 & 4.64 \\
\hline
\end{tabular}


3.3 Dynamic Characteristics for $k=30000 \mathrm{~N} / \mathrm{m}, l=0.48$ $\mathrm{m}$, and $n_{j}=6.8$

Fig. 7 presents results of a computer simulation in which $k=30000 \mathrm{~N} / \mathrm{m}, l=0.48 \mathrm{~m}, n_{j}=6.8$. Here, $k_{x}=$

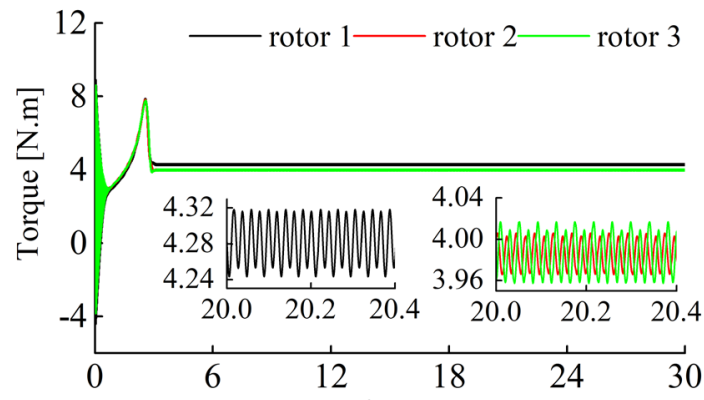

a)
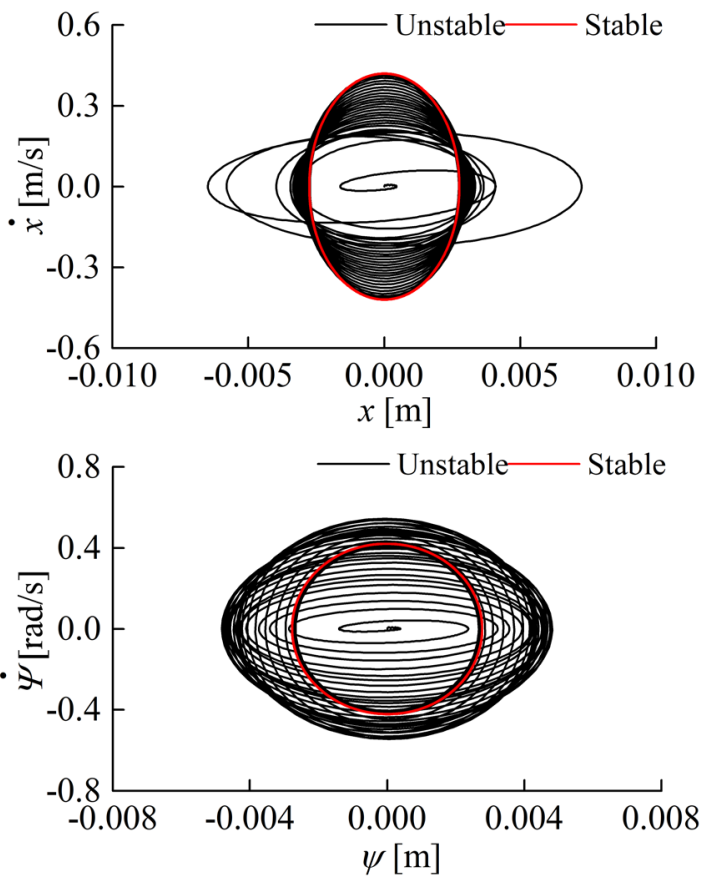

g)

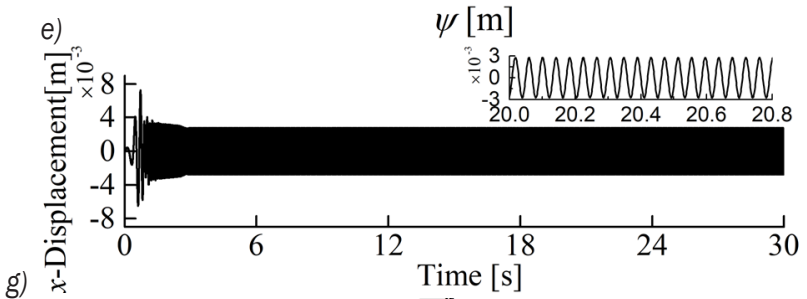

b)
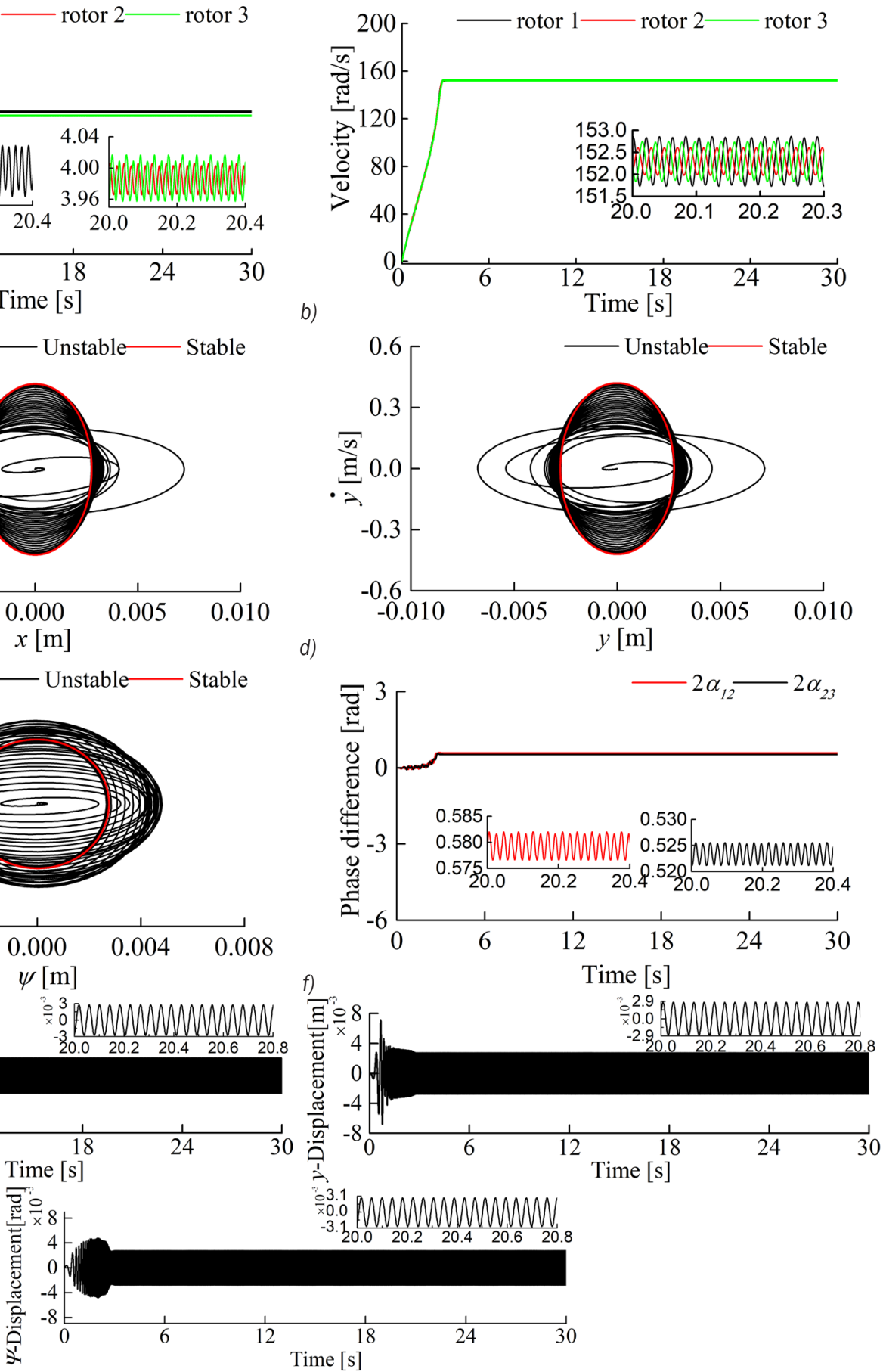

Fig. 7. Simulation results for $k=30000 \mathrm{~N} / \mathrm{m}, l=6.8 \mathrm{~m}, n_{j}=5.48$; a) driving torque of three motors, b) rotational velocity of three motors, c) phase diagram of the system in $x$-direction, d) phase diagram of the system in $y$-direction, e) phase diagram of the system in $\psi$-direction, $f$ ) phase difference of three motors, and g) displacement response of the rigid frame in $x-, y$-, $\psi$-directions, respectively 
respectively. The value of $2 \alpha_{12}$ is $0.58 \mathrm{rad}\left(33.2^{\circ} \triangleq\right.$ $0.58 \mathrm{rad})$, and the value of $2 \alpha_{23}$ is $0.523 \mathrm{rad}\left(29.97^{\circ} \triangleq\right.$ $0.523 \mathrm{rad}$ ). Thus, the simulation results are in good agreement with the numerical results discussed in Figs. 5 and 7 . Fig. $7 \mathrm{~g}$ shows the amplitude of the mass centre of the system in a synchronous state, and its magnitudes are $3 \times 10^{-3} \mathrm{~m}, 2.9 \times 10^{-3} \mathrm{~m}, 3 \times 10^{-3} \mathrm{rad}$, respectively.

\section{EXPERIMENTAL VERIFICATION}

To validate the correctness of above-mentioned theory and numerical analysis, it is necessary to conduct some further corresponding experimental analyses. An experimental strategy with synchronous tests and dynamic tests of the vibrating system is introduced, which consists of high-speed imaging system and dynamic testing system. The experimental prototype, including induction motors (YZS-1.5-4), coupling springs, a rigid frame, an elastic foundation, four supporting springs, etc., are shown in Fig. 8. The motor performance parameters of YZS-1.5-4 are shown in Table 3. Two in-series springs in the coupling springs are always subjected to a changing force alternately in compression when the vibrating system operates at the steady state, which ensures that the three rotors rotating in the same directions are easy to achieve inphase synchronization. The main parameters of the experimental prototypes are $l_{1}=0.41 \mathrm{~m}, l_{2}=0.15 \mathrm{~m}, l_{3}$ $=0.4 \mathrm{~m}, \beta_{1}=159^{\circ}, \beta_{2}=83^{\circ}$, and the other parameters are identical with Table 1. The location parameters of the four measuring points on the prototype are $P_{1}(-0.45 .-0.23), \quad P_{2}(-0.22 .-0.23), \quad P_{3}(0.23 .-0.23)$, $P_{4}(0.46 .-0.23)$, respectively.

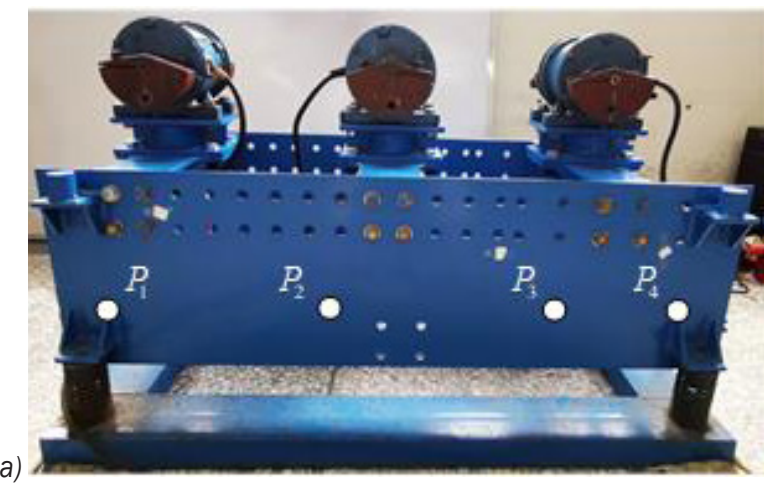

Fig. 8. Experimental prototypes; a) three co-rotating rotors in a vibrating system, b) two co-rotating rotors coupled with two in-series springs

The dynamic testing results of three co-rotating rotors in a vibrating system are shown in Fig. 9. From the spectral analyses shown in Fig. 9a, it can be seen that the peak spectra of point $P_{2}$ and point $P_{3}$ in horizontal and vertical directions reaches a maximum when the system frequency is approximately equal to $24.125 \mathrm{~Hz}$. Figs. 9b and c show acceleration of four measuring points in the horizontal and vertical directions. It can be concluded that their magnitudes are almost the same with a value $24.4 \mathrm{~m} / \mathrm{s}^{2}$. However, the phase constants of the acceleration with points 1 and 4 are different than points 2 and 3 in the horizontal direction, and both the magnitudes and phase constants of their acceleration are different in the vertical direction. Figs. 9d and e show the velocity of four measuring points in horizontal and vertical directions, and Figs. 9f, g, h, and i show the displacement of four measuring points in the horizontal and vertical directions, respectively. The motion trajectories of four measuring point in $x-o-y$ plan are elliptical, as illustrated in Fig. 9j. However, its ovality and vibrating direction on the rigid frame are different, because both the magnitudes and phase constants of their displacements are different in the horizontal and vertical directions. In addition, some simulation results of corresponding experimental prototype are employed to verify the correctness of theoretical analysis based on Eq. (1). The comparison between the dynamic test results and the simulation results with the three co-rotating rotor system are given in Table 4. Those results show that the dynamic test results are proven to be in good agreement with simulation results, and all ranges of error for the

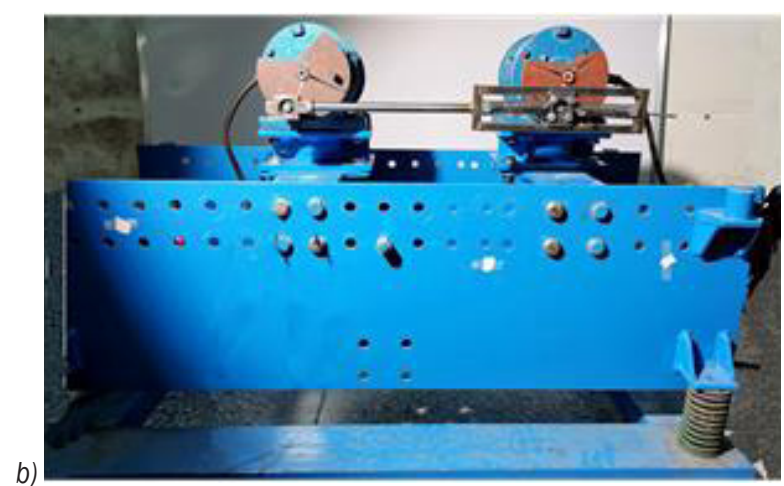

Table 3. Parameters for vibration three-phase asynchronous motor (YZS-1.5-4)

\begin{tabular}{cccccccc}
\hline Parameter & Voltage $[\mathrm{V}]$ & Power rating $[\mathrm{Hz}]$ & Output power $[\mathrm{kW}]$ & Current $[\mathrm{A}]$ & Frequency $[\mathrm{r} / \mathrm{min}]$ & Exciting force $[\mathrm{kN}]$ & Weight $[\mathrm{kg}]$ \\
\hline Value & 380 & 50 & 0.12 & 0.36 & 1500 & 1.5 & 16 \\
\hline
\end{tabular}




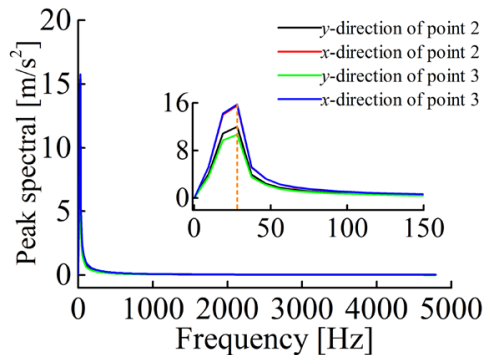

a)

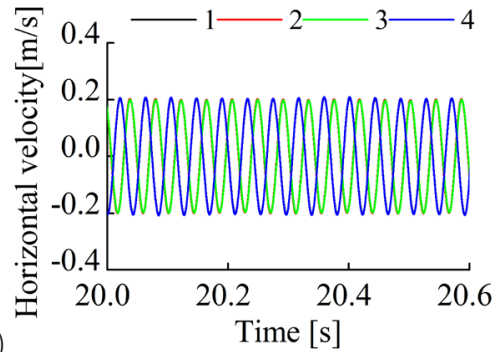

d)

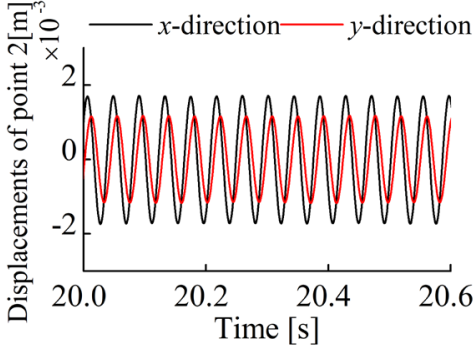

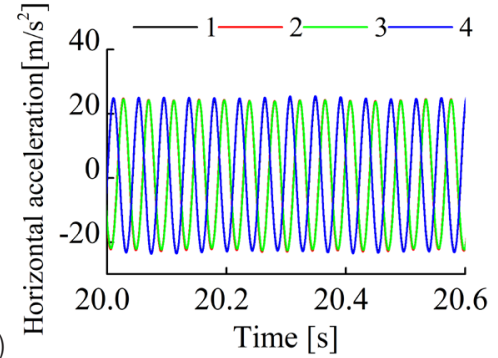

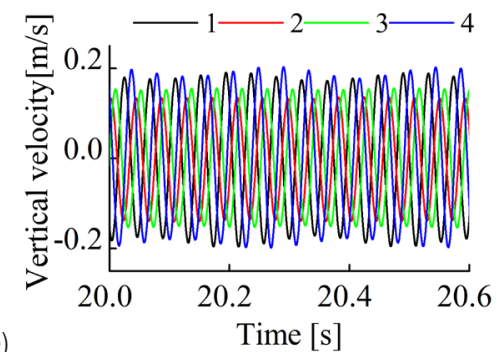

e)

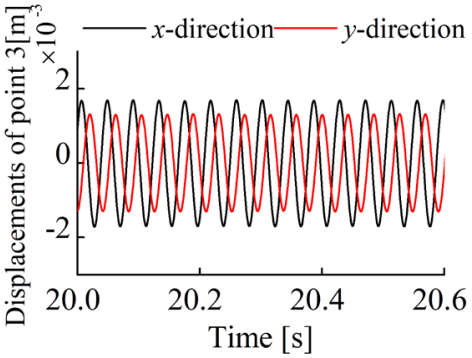

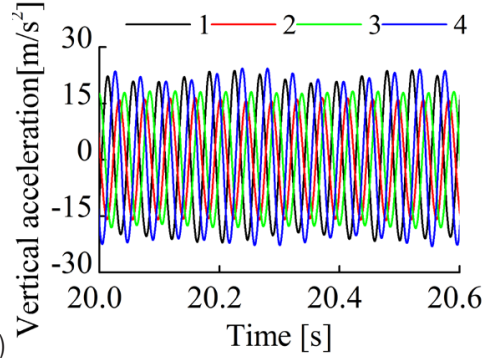
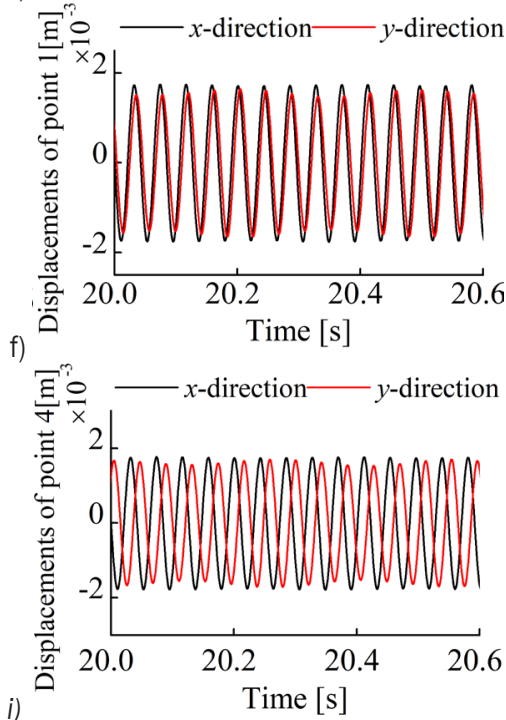

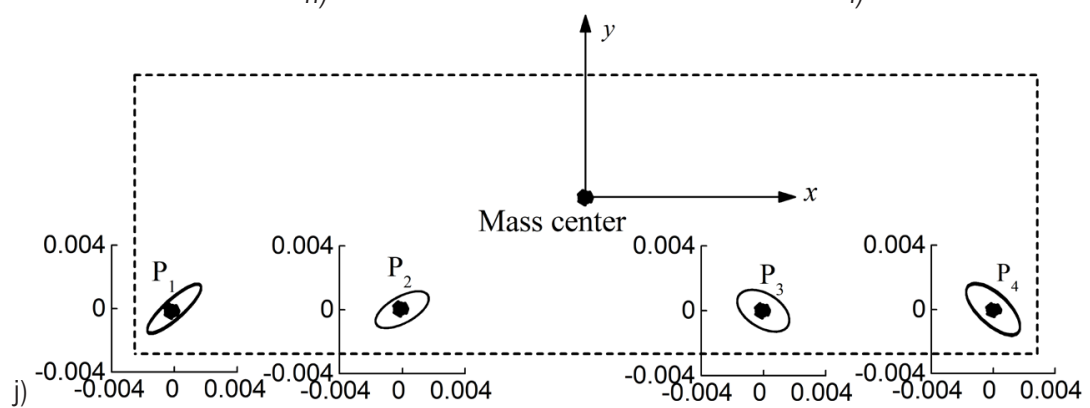

Fig. 9. Dynamic characteristics of three co-rotating rotors system; a) spectral analysis, b) horizontal accelerations of the measuring point, c) vertical accelerations of the measuring point, d) horizontal velocity of the measuring point, e) vertical velocity of the measuring point, f) displacements of the point 1, g) displacements of the point 2, h) displacements of the point 3, I) displacements of the point 4 and j) motion trail of the measuring point in $x-0-y$ plane

Table 4. The amplitude comparison between the dynamic testing results and the simulation results with three co-rotating rotors system

\begin{tabular}{lcccccc}
\hline & \multicolumn{2}{c}{ Results of dynamical testing } & \multicolumn{2}{c}{ Results of dynamical simulation } & \multicolumn{2}{c}{ Error value [\%] } \\
\cline { 2 - 7 } & $x$-direction & $y$-direction & $x$-direction & $y$-direction & $x$-direction & $y$-direction \\
\hline Measuring point $P_{1}$ & 0.0017 & 0.0016 & 0.0015 & 0.0018 & 11.8 & 11.1 \\
\hline Measuring point $P_{2}$ & 0.0017 & 0.0011 & 0.0014 & 0.0015 & 17.6 & 26.7 \\
\hline Measuring point $P_{3}$ & 0.0017 & 0.0013 & 0.0014 & 0.0013 & 17.6 & 16.7 \\
\hline Measuring point $P_{4}$ & 0.0018 & 0.0018 & 0.0015 & 0.0016 & 11.1 \\
\hline
\end{tabular}

measuring-point in the vibrating body are within 30 $\%$.

Fig. 10 shows the transient state of three corotating rotors at different moments. As can be seen those diagrams, the value of $\Delta \alpha_{12}$ is $3.56 \mathrm{rad}$, and the value of $\Delta \alpha_{23}$ is $4.04 \mathrm{rad}$. It is concluded that the synchronous state of any two motors is in anti-phase synchronization when the vibrating system operates 
at the steady state. Compared with the simulation results of corresponding experimental prototype, the simulation values of $\Delta \alpha_{12}$ and $\Delta \alpha_{23}$ are $3.46 \mathrm{rad}, 3.47$ $\mathrm{rad}$, respectively, and their error of magnitudes are within $30 \%$, as shown in Table 5 .

For the experimental prototype with two corotating rotors coupled with springs in a vibrating system, the testing results of dynamic characteristics are shown in Fig. 11. From Figs. 11a and b, it can be seen that the connecting springs with a stress state occurring periodically can be ensured the synchronous operation of the system, the magnitudes of acceleration of point $P_{2}$ and point $P_{3}$ in the horizontal direction are almost the same with a value $20 \mathrm{~m} / \mathrm{s}^{2}$. However, the magnitudes of their acceleration in the vertical direction are more different with values 44.1 $\mathrm{m} / \mathrm{s}^{2}$ and $19.3 \mathrm{~m} / \mathrm{s}^{2}$, respectively. Integrating once and twice for the acceleration during the running process of the steady-state, respectively, we can obtain the velocity and displacement of point $P_{2}$ and point $P_{3}$ in horizontal and vertical directions, as shown in Figs. $11 \mathrm{c}$ to $\mathrm{f}$.

Moreover, comparing simulation results of corresponding parameters, it can be seen that the results of dynamic testing and simulation with two co-rotating rotors coupled with springs are in good agreement, as shown in Table 6. Fig. 11g shows the motion trajectories of point $P_{2}$ and point $P_{3}$ during the running process of the system; it is easily found that its motions are elliptically when the vibrating system

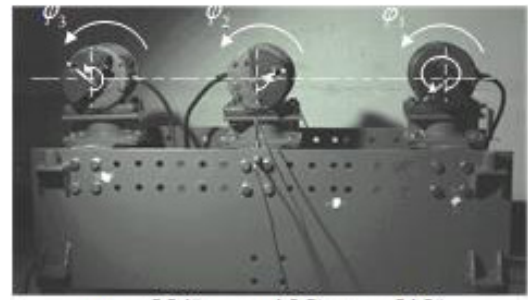

$\varphi_{3} \cong 231, \varphi_{2} \cong 105, \varphi_{1} \cong 312$

$2 \alpha_{12}=\varphi_{1}-\varphi_{2} \cong 207$

$2 \alpha_{23}=\varphi_{2}-\varphi_{3} \cong-126$

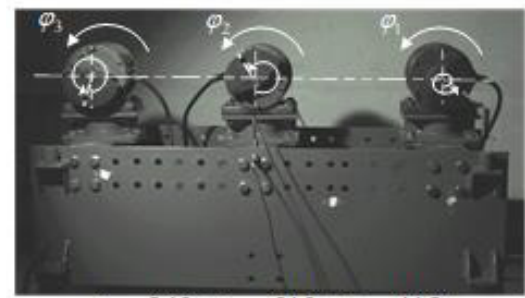

$\varphi_{3} \cong 340^{\circ}, \varphi_{2} \cong 210^{\circ}, \varphi_{1} \cong 415$

$2 \alpha_{12}=\varphi_{1}-\varphi_{2} \cong 205$

$2 \alpha_{23}=\varphi_{2}-\varphi_{3} \cong-130$

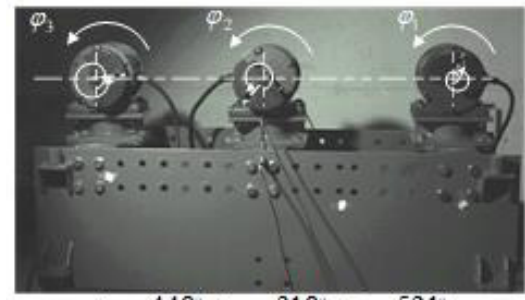

$\varphi_{1} \cong 448^{\circ}, \varphi_{2} \cong 318^{\prime \prime}, \varphi_{1} \cong 521$

$2 \alpha_{12}=\varphi_{1}-\varphi_{2} \cong 203$

$2 \alpha_{23}=\varphi_{2}-\varphi_{3} \cong-130$

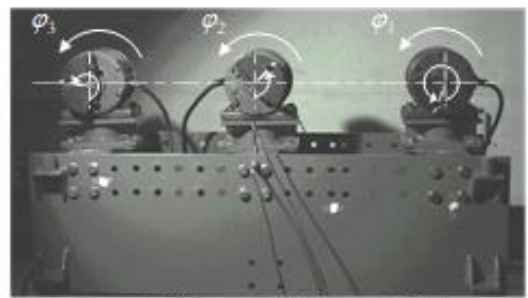

$\varphi_{3} \cong 261^{\prime}, \varphi_{2} \cong 136, \varphi_{1} \cong 341$

$2 \alpha_{12}=\varphi_{1}-\varphi_{2} \cong 205$

$2 \alpha_{23}=\varphi_{2}-\varphi_{3} \cong-125$

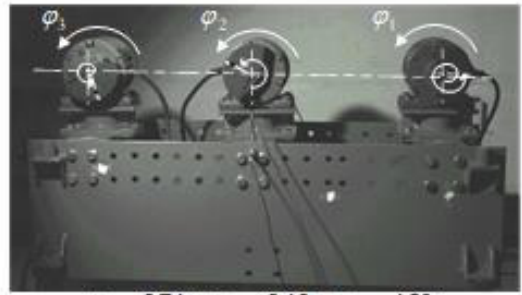

$\varphi_{3} \cong 371^{\prime \prime}, \varphi_{2} \cong 242^{\prime}, \varphi_{1} \cong 450^{\circ}$

$2 \alpha_{12}=\varphi_{1}-\varphi_{2} \cong 208$

$2 \alpha_{23}=\varphi_{2}-\varphi_{3} \cong-129$

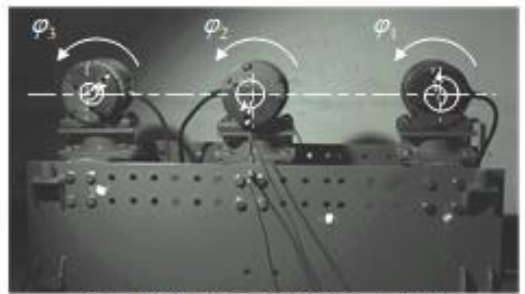

$\varphi_{3} \cong 482^{\prime}, \varphi_{2} \cong 353^{\circ}, \varphi_{1} \cong 557$

$2 \alpha_{12}=\varphi_{1}-\varphi_{2} \cong 204$

$2 \alpha_{23}=\varphi_{2}-\varphi_{3} \cong-129$

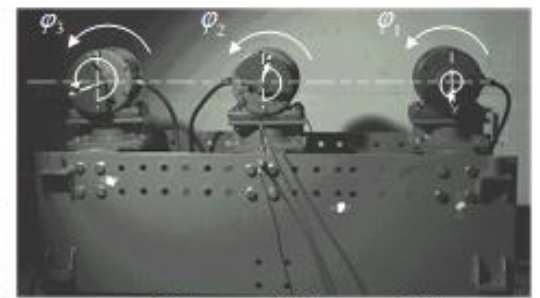

$\varphi_{3} \cong 291^{\prime}, \varphi_{2} \cong 162, \varphi_{1} \cong 365$

$2 \alpha_{12}=\varphi_{1}-\varphi_{2} \cong 203$

$2 \alpha_{23}=\varphi_{2}-\varphi_{3} \cong-129$

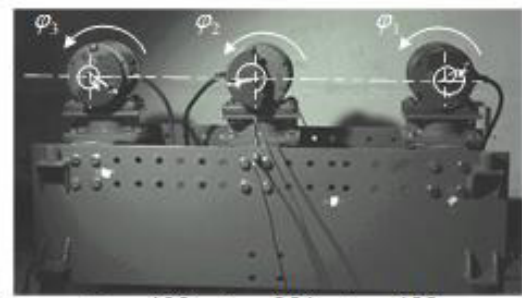

$\varphi_{3} \cong 409^{\circ}, \varphi_{2} \cong 281^{\circ}, \varphi_{1} \cong 483$

$2 \alpha_{12}=\varphi_{1}-\varphi_{2} \cong 202$

$2 \alpha_{23}=\varphi_{2}-\varphi_{3} \cong-128$

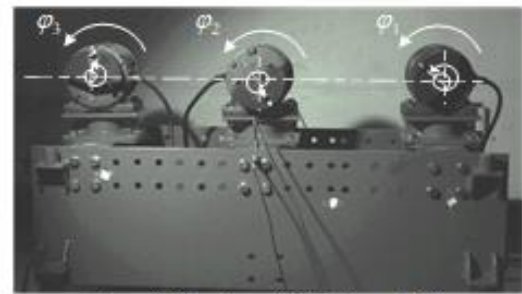

$\varphi_{3} \cong 526^{\prime \prime}, \varphi_{2} \cong 393^{\circ}, \varphi_{1} \cong 594$

$2 \alpha_{12}=\varphi_{1}-\varphi_{2} \cong 201$

$2 \alpha_{23}=\varphi_{2}-\varphi_{3} \cong-133$

Fig. 10. Phase difference with three co-rotating rotors system

Table 5. The comparison between the testing value and the simulation value of phase difference with three co-rotating rotors system

\begin{tabular}{ccccccc}
\hline & \multicolumn{2}{c}{ Experimental test results [rad] } & The result of computer simulation [rad] & \multicolumn{2}{c}{ Error value [\%] } \\
\cline { 2 - 7 } & $2 \alpha_{12}$ & $2 \alpha_{23}$ & $2 \alpha_{12}$ & $2 \alpha_{23}$ & $\Delta \alpha_{12}$ & $\Delta \alpha_{23}$ \\
\hline Phase difference & $204^{\circ} \triangleq 3.56$ & $-128.8^{\circ} \triangleq 4.04$ & 3.46 & 3.47 & 2.8 & 14.1 \\
\hline
\end{tabular}



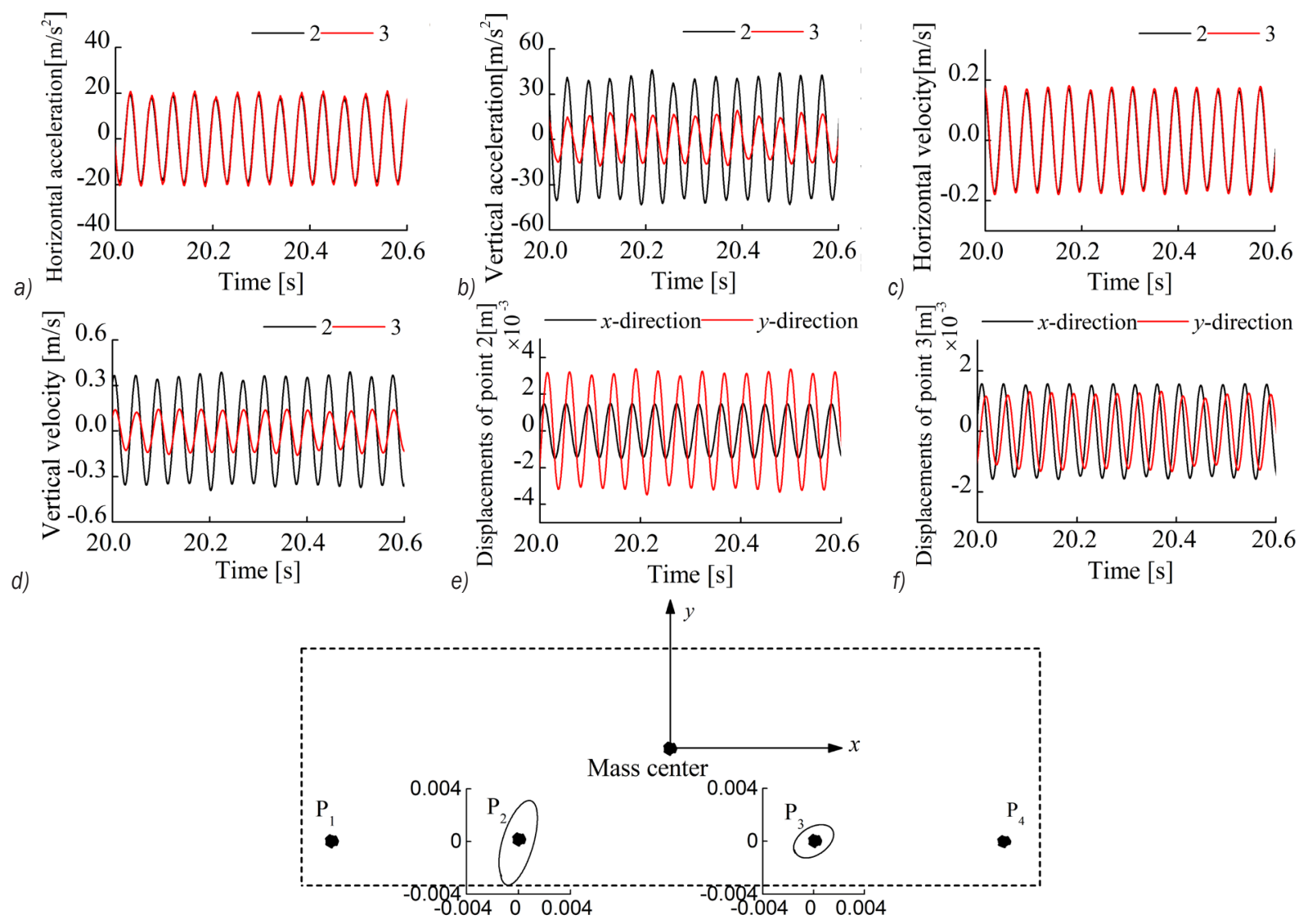

Fig. 11. Dynamic characteristics of two co-rotating rotors coupled with springs in a vibrating system; a) horizontal accelerations of the measuring point, b) vertical accelerations of the measuring point, c) horizontal velocity of the measuring point, $d$ ) vertical velocity of the measuring point, e) displacements of the point $2, f$ ) displacements of the point 3 , and g) motion trail of the measuring point in x-0-y plane

Table 6. The amplitude comparison between the dynamic testing results and the simulation results with two co-rotating rotors coupled with springs in a vibrating system

\begin{tabular}{lcccccc}
\hline & \multicolumn{2}{c}{ Results of dynamical testing } & \multicolumn{2}{c}{ Results of dynamical simulation } & \multicolumn{2}{c}{ Error value [\%] } \\
\cline { 2 - 7 } & $x$-direction & $y$-direction & $x$-direction & $y$-direction & $x$-direction & $y$-direction \\
\hline Measuring point $P_{2}$ & 0.0017 & 0.0016 & 0.0021 & 0.0015 & 19 & 23.8 \\
\hline Measuring point $P_{3}$ & 0.0032 & 0.0014 & 0.0027 & 0.0018 & 15.6 & 22.2 \\
\hline
\end{tabular}

operates at the steady state. However their ovality and vibrating direction on the rigid frame are different.

As can be seen from Fig. 12, the transient state of two co-rotating rotors coupled with springs are presented with an experimental test. Moreover, its comparison between the testing value and the simulation value of phase difference are listed in Table 7. It can be seen that the in-phase synchronization of two co-rotating rotors coupled with springs is easy to implement by the springs suffering from the stress state and unstressed state periodically and alternately. That is to say, the coupling springs can make the phase difference between the three rotors close to zero during the running process of the steady-state. The experimental results are in good agreement with the simulation results in the vibrating system.

\section{CONCLUSION}

In this work, a vibrating mechanism of three corotating rotors system coupled with springs in a nonresonance system is proposed to design large-sized and heavy-duty vibrating screens. The paper is focused on the research of theoretical derivation, numerical analysis, computer simulations, and experimental verification. The conclusions are as follows:

1. For the coupling springs with a small stiffness $k$, the springs have little influence on the 


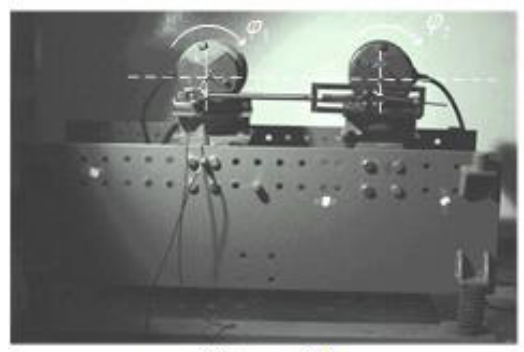

$\varphi_{1} \cong 36, \varphi_{2} \cong 33$

$2 \alpha_{12}=\varphi_{1}-\varphi_{2} \cong 3$

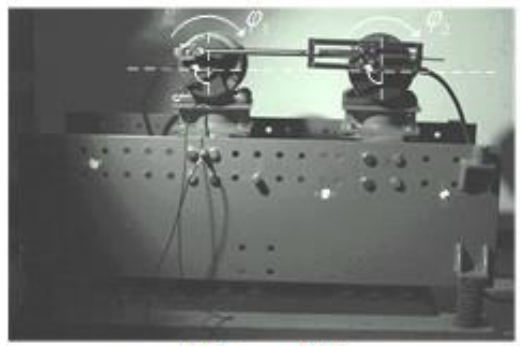

$\varphi_{1} \cong 125, \varphi_{2} \cong 126$

$2 \alpha_{12}=\varphi_{1}-\varphi_{2} \cong-1$

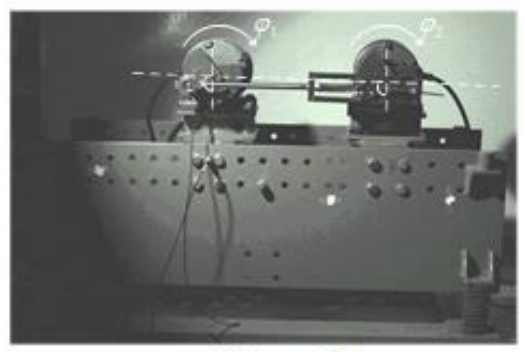

$\varphi_{1} \cong 64^{\prime \prime}, \varphi_{2} \cong 62$

$2 \alpha_{12}=\varphi_{1}-\varphi_{2} \cong 2$

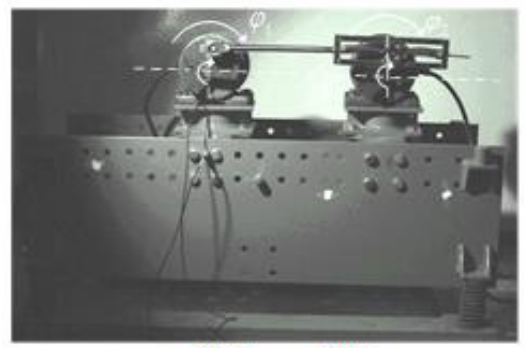

$\varphi_{1} \cong 197, \varphi_{2} \cong 198$

$2 \alpha_{12}=\varphi_{1}-\varphi_{2} \cong-1$

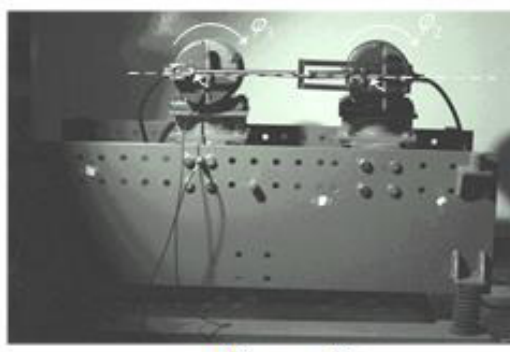

$\varphi_{1} \cong 98^{\circ}, \varphi_{2} \cong 97$

$2 \alpha_{12}=\varphi_{1}-\varphi_{2} \cong 1$

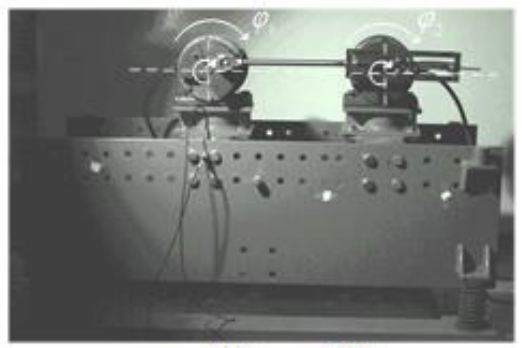

$\varphi_{1} \cong 246^{\circ}, \varphi_{2} \cong 245^{\circ}$

$2 \alpha_{12}=\varphi_{1}-\varphi_{2} \cong 1$

Fig. 12. Phase difference with two co-rotating rotors coupled with springs in a vibrating system

Table 7. The comparison between the testing value and the simulation value of phase difference with two co-rotating rotors coupled with springs in a vibrating system

\begin{tabular}{cccc}
\hline & The results of the indirect experimental tests & The result of computer simulation & Error value \\
\hline Phase difference $2 \alpha_{12}$ & $0.67^{\circ} \triangleq 0.012 \mathrm{rad}$ & $0.75^{\circ} \triangleq 0.013 \mathrm{rad}$ & $7.7 \%$ \\
\hline
\end{tabular}

synchronization characteristics of the vibrating system. Moreover, the synchronous state of motors is always maintained in anti-phase synchronization. However, with the increasing $k$ over a critical value, the phase difference among each two rotors gradually stabilizes at zero. Accordingly, the synchronous state of the system is changed from anti-phase synchronization to in-phase synchronization. Additionally, it can be concluded that the frequency ratio of the system has little influence on synchronous behaviour, but the synchronous state of the system is influenced by positional parameters of three motors, stiffness coefficient of the coupling springs.

2. An electromechanical coupled dynamic model of three co-rotating rotors system coupled with springs is established based on the RungeKutta algorithm with adaptive control. The relationships between the synchronization state of the system and their mechanical-electrical coupling characteristics are investigated. It can be found that the coupling springs with a large enough stiffness can make the phase difference among the three rotors close to zero during the running process of the steady-state. Finally, an experimental prototype including synchronous tests and dynamic tests of the vibrating system is designed to prove to be in good agreement with theory and numerical analysis results.

3. The presented model in this paper can be applied to large-sized and heavy-duty vibrating screens, which can promote the rapid development of new drilling technology and the DFSS towards high capacity, high efficiency, low noise, intelligent, energy-saving, environmental protection, etc.

\section{ACKNOWLEDGEMENTS}

This research was supported by Chengdu International Science and Technology Cooperation Project [No. 2019-GH02-00035-HZ].

\section{NOMENCLATURES}

$m_{i} \quad$ mass of the rotor $i, i=1,2,3,[\mathrm{~kg}]$

$m_{0}$ mass of the rigid frame, $[\mathrm{kg}]$

$r_{i}$ eccentric radius of the rotor $i, i=1,2,3,[\mathrm{~m}]$

$r_{0} \quad$ a standard radius, [m] 
$\varphi_{i}$ angular displacement of the rotor $i, i=1,2,3$, [rad]

$\beta_{i} \quad$ installation angle of the rotor $i, i=1,2,3,\left[^{\circ}\right]$

$l_{i}$ distance from centre of mass to the rotor $i$, $i=1,2,3,[\mathrm{~m}]$

$J_{i} \quad$ rotational inertia of the motor $i, i=1,2,3,\left[\mathrm{~kg} \cdot \mathrm{m}^{2}\right]$

$f_{i} \quad$ damping coefficient of motor $i, i=1,2,3,[\mathrm{~N} \cdot \mathrm{s} / \mathrm{m}]$

$M$ the total mass of the vibrating system, $M=m_{0}+\sum_{1}^{3} m_{i},[\mathrm{~kg}]$

$J$ rotational inertia of the vibrating system, $J=J_{0}+\sum_{1}^{3} m_{i} l_{i}^{2}+\sum_{1}^{3} m_{i} r_{i}^{2},\left[\mathrm{~kg} \cdot \mathrm{m}^{2}\right]$

$f_{j}$ damping constant of the vibrating system in $j-$ direction, $j=x, y, \psi,[\mathrm{N} \cdot \mathrm{s} / \mathrm{m}]$

$k_{j} \quad$ stiffness of four supporting spring in $j$-direction, $j=x, y, \psi,[\mathrm{N} / \mathrm{m}]$

$k$ stiffness coefficient of the connecting spring, $[\mathrm{N} / \mathrm{m}]$

$a$ distance between the rotating centre of motor $i$ and the end of coupling springs, [m]

$\alpha_{12}$ phase differences between motor 1 and motor 2 , [0]

$\alpha_{23}$ phase differences between motor 2 and motor 3 , [0]

$\varepsilon_{i} \quad$ instantaneous change coefficients, $i=1,2,3$,

$\varsigma_{0}, \varsigma_{12}, \varsigma_{23}$ coefficients of the instantaneous change with $\omega_{m}, \alpha_{12}$ and $\alpha_{23}$,

$\varsigma_{x}, \varsigma_{y}, \varsigma_{\psi}$ damping coefficient of the vibrating system in $j$-direction, $j=x, y, \psi, \varsigma_{x}=f_{x} /\left(2 \omega_{n x} M\right), \varsigma_{y}=$ $f_{y} /\left(2 \omega_{n y} M\right), \varsigma_{\psi}=f_{\psi} /\left(2 \omega_{n \psi} M\right)$,

$\omega_{n j}$ natural frequency of the vibrating system in $j$-direction, $j=x, y, \psi, \omega_{n x}=\sqrt{k_{x} / M}$, $\omega_{n y}=\sqrt{k_{y} / M}, \omega_{n \psi}=\sqrt{k_{\psi} / M},[\mathrm{rad} / \mathrm{s}]$

$r_{m}$ dimensionless parameters, $r_{m}=m_{0} / M, i=1,2,3$,

$l_{e}$ dimensionless parameters, $l_{e}=\sqrt{J / M}, i=1,2,3$,

$r_{l i}$ dimensionless parameters, $r_{l i}=l_{i} / l_{e}, i=1,2,3$,

$r_{r i}$ dimensionless parameters, $r_{r i}=r_{i} / r_{0}, i=1,2,3$,

$\eta_{i}$ dimensionless parameters, $\eta_{i}=m_{i} / m_{0}, i=1,2,3$,

$n_{j} \quad$ frequency ratio in in $j$-direction, $j=x, y, \psi$,

$T_{e i}$ driving torque of the rotor $i, i=1,2,3,[\mathrm{~N} \cdot \mathrm{m}]$

$T_{e 0 i}$ output torque of the rotor $i, i=1,2,3,[\mathrm{~N} \cdot \mathrm{m}]$

$\bar{k}_{e 0 i}$ scaling factor of electrical and mechanical damping, $i=1,2,3$,

$T$ total kinetic energy of the system, $[\mathrm{J}]$

$V$ total potential energy of the system, $[\mathrm{J}]$

$Q_{i}$ generalized force of the system

$q_{i} \quad$ generalized coordinate of the system

$T_{z}$ the sum of kinetic energy with three motors, [J]

$\langle\bar{\bullet}\rangle$ integrating over one period $T$ of time, $\langle\bar{\bullet}\rangle=\int_{T}^{t_{0}+T}(\bullet) d t$

$(\bullet)$ The first derivative of time, $d(\bullet) / d t$

(•) The second derivative of time, $d^{2}(\bullet) / d t^{2}$

\section{REFERENCES}

[1] Baragetti, S. (2015). Innovative structural solution for heavy loaded vibrating screens. Minerals Engineering, vol. 84, p. 1526, D0I:10.1016/j.mineng.2015.09.011.

[2] Dong, K., Esfandiary, A.H., Yu, A.B. (2017). Discrete particle simulation of particle flow and separation on a vibrating screen: Effect of aperture shape. Powder Technology, vol. 314, p. 195-202, D0l:10.1016/j.powtec.2016.11.004.

[3] Dong, K.J., Yu, A.B. (2012). Numerical simulation of the particle flow and sieving behaviour on sieve bend/low head screen combination. Minerals Engineering, vol. 31, no. 4, p. 2-9, Dol:10.1016/j.mineng.2011.10.020.

[4] Blekhman, I.I. (1988). Synchronization in Science and Technology. ASME Press, New York.

[5] Blekhman, I.I., Sorokin, V.S. (2018). On a "deterministic" explanation of the stochastic resonance phenomenon. Nonlinear Dynamics, vol. 93, p. 767-778, D0l:10.1007/ s11071-018-4225-y.

[6] Blekhman, I.I., Sorokin, V.S. (2010). On the separation of fast and slow motions in mechanical systems with high-frequency modulation of the dissipation coefficient. Journal of Sound and Vibration, vol. 329, no. 23, p. 4936-4949, D0I:10.1016/j. jsv.2010.06.008.

[7] Balthazar, J.M., Felix, J.L.P., Brasil, R.M.L.R.F. (2005). Some comments on the numerical simulation of self-synchronization of four non-ideal exciters. Applied Mathematics \& Computation, vol. 164, no. 2, p. 615-625, D0l:10.1016/j. amc.2004.06.010.

[8] Balthazar, J.M., Felix, J.L.P., Brasil, R.M.L.R.F. (2016). Short comments on self-synchronization of two non-ideal sources supported by a flexible portal frame structure. Journal of Vibration and Control, vol. 10, no. 12, p. 1739-1748, DOI:10.1177/1077546304041754.

[9] Wen, B., Fan, J., Zhao, C., Xiong, W. (2012). Vibratory and Controlled Synchronization Engineering, Alpha Science, Paris.

[10] Wen, B., Zhang, H., Liu, S., He, Q., Zhao, C. (2010). Theory and Techniques of Vibrating Machinery and Their Applications. Science Press, Beijing.

[11] Zhang, X.L., Wen, B.C., Zhao, C.Y. (2013). Synchronization of three non-identical coupled exciters with the same rotating directions in a far-resonant vibrating system. Journal of Sound and Vibration, vol. 332, no. 9, p. 2300-2317, D0I:10.1016/j. jsv.2012.12.003.

[12] Zhang, X., Wen, B., Zhao, C. (2014). Vibratory synchronization transmission of two exciters in a super-resonant vibrating system. Journal of Mechanical Science and Technology, vol. 28, p. 2049-2058, D0I:10.1007/s12206-014-0108-4.

[13] Fang, P., Hou, Y.J. (2018). Synchronization characteristics of a rotor-pendula system in multiple coupling resonant systems. Proceedings of the Institution of Mechanical Engineers, Part C: Journal of Mechanical Engineering Science, vol. 232, no. 10, p. 1802-1822, Dol:10.1177/0954406217711468.

[14] Fang, P., Hou, Y.J., Du, M.J. (2019). Synchronization behavior of triple-rotor-pendula system in a dual-superfar resonance system. Proceedings of the Institution of Mechanical Engineers, Part C: Journal of Mechanical 
Engineering Science, vol. 233, no. 5, p. 1620-1640, DOI:10.1177/0954406218774585.

[15] Chen, X., Kong, X., Liu, Y., Wen, B. (2016). Synchronization and coupling dynamic characteristics of a dual-rotors exciter. Journal of Vibroengineering, vol. 18, no. 5, p. 3318-3328, DOl:10.21595/jve.2016.16918.

[16] Chen, X.Z., Kong, X.X., Zhang, X.L., Li, L.X., Wen, B.C. (2016). On the synchronization of two eccentric rotors with common rotational axis: Theory and experiment. Shock and Vibration, vol. 2016, art. ID 6973597, DOl:10.1155/2016/6973597.

[17] Huang, Z.L., Song, G.Q., Li, Y.M., Sun, M.N. (2019). Synchronous control of two counter-rotating eccentric rotors in nonlinear coupling vibration system. Mechanical Systems and Signal Processing, vol. 114, p. 68-83, D0l:10.1016/j. ymssp.2018.05.006.

[18] Huang, Z., Song, G., Zhang, Z., Zhang, X. (2019). Control synchronization of two nonidentical homodromy exciters in nonlinear coupled vibration system. IEEE Access, vol. 7, p. 109934-109944, D0I:10.1109/access.2019.2933033.

[19] Kong, X.X., Chen, C.Z., Wen, B.C. (2018). Composite synchronization of three eccentric rotors driven by induction motors in a vibrating system. Mechanical Systems and Signal Processing, vol. 102, p. 158-179, D0l:10.1016/j. ymssp.2017.09.025.

[20] Kong, X.X., Zhang, X.L., Chen, X.Z., Wen, B.C., Wang, B. (2016). Phase and speed synchronization control of four eccentric rotors driven by induction motors in a linear vibratory feeder with unknown time-varying load torques using adaptive sliding mode control algorithm. Journal of Sound and Vibration, vol. 370, p. 23-42, D0l:10.1016/j.jsv.2016.02.013.

[21] Huang, Z.L., Li, Y.M., Song, G.Q., Zhang, X.L., Zhang, Z.C. (2019). Speed and phase adjacent cross-coupling synchronous control of multi-exciters in vibration system considering material influence. IEEE Access, vol. 7, p. 6320463216, D0l:10.1109/Access.2019.2916983.

[22] Zhang, X., Wen, B., Zhao, C. (2014). Vibratory synchronization and coupling dynamic characteristics of multiple unbalanced rotors on a mass-spring rigid base. International Journal of Non-Linear Mechanics, vol. 60, p. 1-8, D0l:10.1016/j. ijnonlinmec.2013.12.002.

[23] Hou, Y. (2007). The synchronism theory of three motor selfsynchronism exciting elliptical motion shaker. Journal of Southwest Petroleum University, vol. 29, p. 168-172.

[24] Wang,C., Liu, J., Luo, Z. (2019). Suppression of self-excited vibrations in rotating machinery utilizing leaf springs. Strojniški vestnik - Journal of Mechanical Engineering, vol. 65,P. 599608, D0l:10.5545/sv-jme.2019.6112.

[25] Si, H., Cao, L., Li, P. (2020). Dynamic characteristics and stability prediction of steam turbine rotor based on mesh deformation. Strojniški vestnik - Journal of Mechanical Engineering, vol. 66, p. 164-174, D0l:10.5545/svjme.2019.6283.

\section{APPENDIX}

$$
\begin{aligned}
& \rho_{11}=\frac{J_{1}}{m_{0} r_{0}^{2}}+\frac{1}{2} \eta_{1}^{2} r_{r 1}^{2} W_{c 1}, \quad \rho_{12}=\frac{J_{2}}{m_{0} r_{0}^{2}}+\frac{1}{2} \eta_{2} r_{r 2} \eta_{2} r_{r 2} W_{c 2}, \quad \rho_{13}=\frac{J_{3}}{m_{0} r_{0}^{2}}+\frac{1}{2} \eta_{3} r_{r 3} \eta_{3} r_{r 3} W_{c 3}, \\
& \chi_{12}^{\prime}=\frac{1}{2} \eta_{1} \eta_{2} r_{r 1} r_{r 2}\left[W_{c 12} \cos \left(\alpha_{12}+\theta_{c 12}\right)-W_{s 12} \sin \left(\alpha_{12}+\theta_{s 12}\right)\right], \quad \chi_{13}^{\prime}=\frac{1}{2} \eta_{1} \eta_{3} r_{r 1} r_{r 3}\left[W_{c 13} \cos \left(\alpha_{12}+\alpha_{23}+\theta_{c 13}\right)-W_{s 13} \sin \left(\alpha_{12}+\alpha_{23}+\theta_{s 13}\right)\right], \\
& k_{11}=\frac{\bar{k}_{e 01}}{m_{0} r_{0}{ }^{2} \omega_{m}{ }^{2}}+\frac{f_{1}}{m_{0} r_{0}{ }^{2} \omega_{m}}+\eta_{1}{ }^{2} r_{r 1}{ }^{2} W_{s 1}, \quad k_{22}=\frac{\bar{k}_{e 02}}{m_{0} r_{0}{ }^{2} \omega_{m}{ }^{2}}+\frac{f_{2}}{m_{0} r_{0}{ }^{2} \omega_{m}}+\eta_{2}{ }^{2} r_{r 2}{ }^{2} W_{s 2}, \quad k_{33}=\frac{\bar{k}_{e 03}}{m_{0} r_{0}{ }^{2} \omega_{m}{ }^{2}}+\frac{f_{3}}{m_{0} r_{0}{ }^{2} \omega_{m}}+\eta_{3} r_{r 3} \eta_{3} r_{r 3} W_{s 3}, \\
& \chi_{12}=\eta_{1} r_{r 1} \eta_{2} r_{r 2}\left[W_{c 12} \sin \left(\alpha_{12}+\theta_{c 12}\right)+W_{s 12} \cos \left(\alpha_{12}+\theta_{s 12}\right)\right], \quad \chi_{13}=\eta_{1} r_{r 1} \eta_{3} r_{r 3}\left[W_{c 13} \sin \left(\alpha_{12}+\alpha_{23}+\theta_{c 13}\right)+W_{s 13} \sin \left(\alpha_{12}+\alpha_{23}+\theta_{s 13}\right)\right], \\
& \mu_{1}=\frac{T_{e 01}}{m_{0} r_{0}^{2} \omega_{m}}-\frac{f_{1}}{m_{0} r_{0}^{2}}-\frac{1}{2} \eta_{1} r_{r 1} \eta_{1} r_{r 1} \omega_{m} W_{s 1}-\frac{1}{2} \eta_{1} r_{r 1} \eta_{2} r_{r 2} \omega_{m}\left[W_{c 12} \sin \left(\alpha_{12}+\theta_{c 12}\right)+W_{s 12} \cos \left(\alpha_{12}+\theta_{s 12}\right)\right] \\
& -\frac{1}{2} \eta_{1} r_{r 1} \eta_{3} r_{r 3} \omega_{m}\left[W_{c 13} \sin \left(\alpha_{12}+\alpha_{23}+\theta_{c 13}\right)+W_{s 13} \sin \left(\alpha_{12}+\alpha_{23}+\theta_{s 13}\right)\right]-\frac{k a^{2}}{m_{0} r_{0}^{2} \omega_{m}} \sin \alpha_{12}+\frac{F_{1}}{m_{0} r_{0}^{2}}, \\
& \chi_{21}^{\prime}=\frac{1}{2} \eta_{2} r_{r 2} \eta_{1} r_{r 1}\left[W_{c 12} \cos \left(\alpha_{12}+\theta_{c 12}\right)+W_{s 12} \sin \left(\alpha_{12}+\theta_{s 12}\right)\right], \quad \chi_{23}^{\prime}=\frac{1}{2} \eta_{2} r_{r 2} \eta_{3} r_{r 3}\left[W_{c 23} \cos \left(\alpha_{23}+\theta_{c 23}\right)-W_{s 23} \sin \left(\alpha_{23}+\theta_{s 23}\right)\right], \\
& \chi_{21}=-\eta_{2} r_{r 2} \eta_{1} r_{r 1}\left[W_{c 12} \sin \left(\alpha_{12}+\theta_{c 12}\right)-W_{s 12} \cos \left(\alpha_{12}+\theta_{s 12}\right)\right], \quad \chi_{23}=\eta_{2} \eta_{3} r_{r 2} r_{r 3}\left[W_{c 23} \sin \left(\alpha_{23}+\theta_{c 23}\right)+W_{s 23} \cos \left(\alpha_{23}+\theta_{s 23}\right)\right] \text {, } \\
& \mu_{2}=\frac{T_{e 02}}{m_{0} r_{0}{ }^{2} \omega_{m}}-\frac{f_{2}}{m_{0} r_{0}{ }^{2}}+\frac{1}{2} \eta_{1} \eta_{2} r_{r 1} r_{r 2} \omega_{m}\left[W_{c 12} \sin \left(\alpha_{12}+\theta_{c 12}\right)-W_{s 12} \cos \left(\alpha_{12}+\theta_{s 12}\right)\right]-\frac{1}{2} \eta_{2}{ }^{2} r_{r 2}{ }^{2} \omega_{m} W_{s 2} \\
& -\frac{1}{2} \eta_{2} \eta_{3} r_{r 2} r_{r 3} \omega_{m}\left[W_{c 23} \sin \left(\alpha_{23}+\theta_{c 23}\right)+W_{s 23} \cos \left(\alpha_{23}+\theta_{s 23}\right)\right]+\frac{k a^{2}}{m_{0} r_{0}{ }^{2} \omega_{m}}\left(\sin \alpha_{12}-\sin \alpha_{23}\right)+\frac{F_{2}}{m_{0} r_{0}{ }^{2}}, \\
& \chi_{31}^{\prime}=\frac{1}{2} \eta_{3} r_{r 3} \eta_{1} r_{r 1}\left[W_{c 13} \cos \left(\alpha_{12}+\alpha_{23}+\theta_{c 13}\right)+W_{s 13} \sin \left(\alpha_{12}+\alpha_{23}+\theta_{s 13}\right)\right], \quad \chi_{32}^{\prime}=\frac{1}{2} \eta_{3} r_{r 3} \eta_{2} r_{r 2}\left[W_{c 23} \cos \left(\alpha_{23}+\theta_{c 23}\right)+W_{s 23} \sin \left(\alpha_{23}+\theta_{s 23}\right)\right], \\
& \chi_{31}=-\eta_{3} r_{r 3} \eta_{1} r_{r 1}\left[W_{c 13} \sin \left(\alpha_{12}+\alpha_{23}+\theta_{c 13}\right)-W_{s 13} \cos \left(\alpha_{12}+\alpha_{23}+\theta_{s 13}\right)\right], \quad \chi_{32}=-\eta_{3} r_{r 3} \eta_{2} r_{r 2}\left[W_{c 23} \sin \left(\alpha_{23}+\theta_{c 23}\right)-W_{s 23} \cos \left(\alpha_{23}+\theta_{s 23}\right)\right] \text {, } \\
& \mu_{3}=\frac{T_{e 03}}{m_{0} r_{0}^{2} \omega_{m}}-\frac{f_{3}}{m_{0} r_{0}^{2}}+\frac{1}{2} \eta_{3} r_{r 3} \eta_{1} r_{r 1} \omega_{m}\left[W_{c 13} \sin \left(\alpha_{12}+\alpha_{23}+\theta_{c 13}\right)-W_{s 13} \cos \left(\alpha_{12}+\alpha_{23}+\theta_{s 13}\right)\right] \\
& +\frac{1}{2} \eta_{3} r_{r 3} \eta_{2} r_{r 2} \omega_{m}\left[W_{c 23} \sin \left(\alpha_{23}+\theta_{c 23}\right)-W_{s 23} \cos \left(\alpha_{23}+\theta_{s 23}\right)\right]-\frac{1}{2} \eta_{3} r_{r 3} \eta_{3} r_{r 3} \omega_{m} W_{s 3}+\frac{k a^{2}}{m_{0} r_{0}^{2} \omega_{m}} \sin \alpha_{23}+\frac{F_{3}}{m_{0} r_{0}^{2}},
\end{aligned}
$$


$W_{c 1}=r_{m}\left[\mu_{x} \cos \gamma_{x}+\mu_{y} \cos \gamma_{y}+\mu_{\psi} r_{l 1}{ }^{2} \cos \gamma_{\psi}\right], \quad W_{c 2}=r_{m}\left[\mu_{x} \cos \gamma_{x}+\mu_{y} \cos \gamma_{y}+\mu_{\psi} r_{l 2}{ }^{2} \cos \gamma_{\psi}\right]$,

$W_{c 3}=r_{m}\left[\mu_{x} \cos \gamma_{x}+\mu_{y} \cos \gamma_{y}+\mu_{\psi} r_{l 3}^{2} \cos \gamma_{\psi}\right]$,

$W_{s 1}=r_{m}\left[\mu_{x} \sin \gamma_{x}+\mu_{y} \sin \gamma_{y}+\mu_{\psi} r_{l 1}^{2} \sin \gamma_{\psi}\right], \quad W_{s 2}=r_{m}\left[\mu_{x} \sin \gamma_{x}+\mu_{y} \sin \gamma_{y}+\mu_{\psi} r_{l 2}^{2} \sin \gamma_{\psi}\right]$,

$W_{s 3}=r_{m}\left[\mu_{x} \sin \gamma_{x}+\mu_{y} \sin \gamma_{y}+\mu_{\psi} r_{l 3}^{2} \sin \gamma_{\psi}\right]$,

$a_{s 12}=\mu_{x} \sin \gamma_{x}+\mu_{y} \sin \gamma_{y}+\mu_{\psi \psi} r_{l 1} r_{l 2} \sin \gamma_{\psi} \cos \left(\beta_{1}-\beta_{2}\right), \quad b_{s 12}=\mu_{\psi} r_{l 1} r_{l 2} \sin \gamma_{\psi} \sin \left(\beta_{1}-\beta_{2}\right)$,

$W_{s 12}=r_{m} \sqrt{a_{s 12}{ }^{2}+b_{s 12}{ }^{2}}, \quad \theta_{s 12}=\left\{\begin{array}{ll}\arctan \left(b_{s 12} / a_{s 12}\right) & a_{s 12} \geq 0 \\ \pi+\arctan \left(b_{s 12} / a_{s 12}\right) & a_{s 12}<0\end{array}\right.$,

$a_{s 13}=\mu_{x} \sigma \sin \gamma_{x}+\mu_{y} \sin \gamma_{y}+\mu_{\psi} r_{l 1} r_{l 3} \sin \gamma_{\psi} \cos \left(\beta_{1}-\sigma \beta_{3}\right), \quad b_{s 13}=\mu_{\psi} r_{l 1} r_{l 3} \sin \gamma_{\psi} \sin \left(\beta_{1}-\sigma \beta_{3}\right)$,

$W_{s 13}=r_{m} \sqrt{a_{s 13}{ }^{2}+b_{s 13}{ }^{2}}, \quad \theta_{s 13}=\left\{\begin{array}{ll}\arctan \left(b_{s 13} / a_{s 13}\right) & a_{s 13} \geq 0 \\ \pi+\arctan \left(b_{s 13} / a_{s 13}\right) & a_{s 13}<0\end{array}\right.$,

$a_{s 23}=\mu_{x} \sigma \sin \gamma_{x}+\mu_{y} \sin \gamma_{y}+\mu_{\psi} r_{l 2} r_{l 3} \sin \gamma_{\psi} \cos \left(\beta_{2}-\sigma \beta_{3}\right), \quad b_{s 23}=\mu_{\psi} r_{l 2} r_{l 3} \sin \gamma_{\psi} \sin \left(\beta_{2}-\sigma \beta_{3}\right)$,

$W_{s 23}=r_{m} \sqrt{a_{s 23}^{2}+b_{s 23}^{2}}, \quad \theta_{s 23}=\left\{\begin{array}{ll}\arctan \left(b_{s 23} / a_{s 23}\right) & a_{s 23} \geq 0 \\ \pi+\arctan \left(b_{s 23} / a_{s 23}\right) & a_{s 23}<0\end{array}\right.$,

$a_{c 12}=\mu_{x} \cos \gamma_{x}+\mu_{y} \cos \gamma_{y}+\mu_{\psi} r_{l 1} r_{l 2} \cos \gamma_{\psi} \cos \left(\beta_{1}-\beta_{2}\right), \quad b_{c 12}=\mu_{\psi} r_{l 1} r_{l 2} \cos \gamma_{\psi} \sin \left(\beta_{1}-\beta_{2}\right)$

$W_{c 12}=r_{m} \sqrt{a_{c 12}{ }^{2}+b_{c 12}{ }^{2}}, \quad \theta_{c 12}=\left\{\begin{array}{ll}\arctan \left(b_{c 12} / a_{c 12}\right) & a_{c 12} \geq 0 \\ \pi+\arctan \left(b_{c 12} / a_{c 12}\right) & a_{c 12}<0\end{array}\right.$,

$a_{c 13}=\mu_{x} \sigma \cos \gamma_{x}+\mu_{y} \cos \gamma_{y}+\mu_{\psi} r_{l 1} r_{l 3} \cos \gamma_{\psi} \cos \left(\beta_{1}-\sigma \beta_{3}\right), \quad b_{c 13}=\mu_{\psi \psi} r_{l 1} r_{l 3} \cos \gamma_{\psi} \sin \left(\beta_{1}-\sigma \beta_{3}\right)$,

$W_{c 13}=r_{m} \sqrt{a_{c 13}{ }^{2}+b_{c 13}{ }^{2}}, \quad \theta_{c 13}=\left\{\begin{array}{ll}\arctan \left(b_{c 13} / a_{c 13}\right) & a_{c 13} \geq 0 \\ \pi+\arctan \left(b_{c 13} / a_{c 13}\right) & a_{c 13}<0\end{array}\right.$,

$a_{c 23}=\mu_{x} \sigma \cos \gamma_{x}+\mu_{y} \cos \gamma_{y}+\mu_{\psi} r_{l 2} r_{l 3} \cos \gamma_{\psi} \cos \left(\beta_{2}-\sigma \beta_{3}\right), \quad b_{c 23}=\mu_{\psi} r_{l 2} r_{l 3} \cos \gamma_{\psi} \sin \left(\beta_{2}-\sigma \beta_{3}\right)$,

$W_{c 23}=r_{m} \sqrt{a_{c 23}{ }^{2}+b_{c 23}{ }^{2}}, \quad \theta_{c 23}=\left\{\begin{array}{ll}\arctan \left(b_{c 23} / a_{c 23}\right) & a_{c 23} \geq 0 \\ \pi+\arctan \left(b_{c 23} / a_{c 23}\right) & a_{c 23}<0\end{array}\right.$,

$f_{1}\left(k, \varphi_{1}, \varphi_{2}, \varphi_{3}, \beta, l, a\right)=\frac{-a^{2} \sin \left(\varphi_{2}-\varphi_{1}\right)+l a \cos \varphi_{1} \cos \beta}{\sqrt{l^{2} \cos ^{2} \beta+2 a^{2}-2 a^{2} \cos \left(\varphi_{2}-\varphi_{1}\right)+2 l a \sin \varphi_{1} \cos \beta-2 l a \sin \varphi_{2} \cos \beta}}$,

$f_{2}\left(k, \varphi_{1}, \varphi_{2}, \varphi_{3}, \beta, l, a\right)=\frac{a^{2} \sin \left(\varphi_{2}-\varphi_{1}\right)-l a \cos \varphi_{2} \cos \beta}{\sqrt{l^{2} \cos ^{2} \beta+2 a^{2}-2 a^{2} \cos \left(\varphi_{2}-\varphi_{1}\right)+2 l a \sin \varphi_{1} \cos \beta-2 l a \sin \varphi_{2} \cos \beta}}$

$+\frac{-a^{2} \sin \left(\varphi_{3}-\varphi_{2}\right)+l a \cos \varphi_{2} \cos \beta}{\sqrt{l^{2} \cos ^{2} \beta+2 a^{2}-2 a^{2} \cos \left(\varphi_{3}-\varphi_{2}\right)+2 l a \sin \varphi_{2} \cos \beta-2 l a \sin \varphi_{3} \cos \beta}}$,

$f_{3}\left(k, \varphi_{1}, \varphi_{2}, \varphi_{3}, \beta, l, a\right)=\frac{a^{2} \sin \left(\varphi_{3}-\varphi_{2}\right)-l a \cos \varphi_{3} \cos \beta}{\sqrt{l^{2} \cos ^{2} \beta+2 a^{2}-2 a^{2} \cos \left(\varphi_{3}-\varphi_{2}\right)+2 l a \sin \varphi_{2} \cos \beta-2 l a \sin \varphi_{3} \cos \beta}}$,

$F_{1}=\frac{1}{2 \pi} \int_{0}^{2 \pi} f_{1}\left(k, \varphi_{1}, \varphi_{2}, \varphi_{3}, \beta, l, a\right) d \varphi, \quad F_{2}=\frac{1}{2 \pi} \int_{0}^{2 \pi} f_{2}\left(k, \varphi_{1}, \varphi_{2}, \varphi_{3}, \beta, l, a\right) d \varphi, \quad F_{3}=\frac{1}{2 \pi} \int_{0}^{2 \pi} f_{3}\left(k, \varphi_{1}, \varphi_{2}, \varphi_{3}, \beta, l, a\right) d \varphi$,

$f_{1}\left(k, \varphi_{1}, \varphi_{2}, \varphi_{3}, \beta, l, a\right)=\frac{-a^{2} \sin \left(\varphi_{2}-\varphi_{1}\right)+l a \cos \varphi_{1} \cos \beta}{\sqrt{l^{2} \cos ^{2} \beta+2 a^{2}-2 a^{2} \cos \left(\varphi_{2}-\varphi_{1}\right)+2 l a \sin \varphi_{1} \cos \beta-2 l a \sin \varphi_{2} \cos \beta}}$,

$f_{2}\left(k, \varphi_{1}, \varphi_{2}, \varphi_{3}, \beta, l, a\right)=\frac{a^{2} \sin \left(\varphi_{2}-\varphi_{1}\right)-l a \cos \varphi_{2} \cos \beta}{\sqrt{l^{2} \cos ^{2} \beta+2 a^{2}-2 a^{2} \cos \left(\varphi_{2}-\varphi_{1}\right)+2 l a \sin \varphi_{1} \cos \beta-2 l a \sin \varphi_{2} \cos \beta}}$

$$
+\frac{-a^{2} \sin \left(\varphi_{3}-\varphi_{2}\right)+l a \cos \varphi_{2} \cos \beta}{\sqrt{l^{2} \cos ^{2} \beta+2 a^{2}-2 a^{2} \cos \left(\varphi_{3}-\varphi_{2}\right)+2 l a \sin \varphi_{2} \cos \beta-2 l a \sin \varphi_{3} \cos \beta}},
$$

$f_{3}\left(k, \varphi_{1}, \varphi_{2}, \varphi_{3}, \beta, l, a\right)=\frac{a^{2} \sin \left(\varphi_{3}-\varphi_{2}\right)-l a \cos \varphi_{3} \cos \beta}{\sqrt{l^{2} \cos ^{2} \beta+2 a^{2}-2 a^{2} \cos \left(\varphi_{3}-\varphi_{2}\right)+2 l a \sin \varphi_{2} \cos \beta-2 l a \sin \varphi_{3} \cos \beta}}$,

$F_{1}=\frac{1}{2 \pi} \int_{0}^{2 \pi} f_{1}\left(k, \varphi_{1}, \varphi_{2}, \varphi_{3}, \beta, l, a\right) d \varphi, \quad F_{2}=\frac{1}{2 \pi} \int_{0}^{2 \pi} f_{2}\left(k, \varphi_{1}, \varphi_{2}, \varphi_{3}, \beta, l, a\right) d \varphi, \quad F_{3}=\frac{1}{2 \pi} \int_{0}^{2 \pi} f_{3}\left(k, \varphi_{1}, \varphi_{2}, \varphi_{3}, \beta, l, a\right) d \varphi$, 